\title{
Intramolecular energy transfer and the driving mechanisms for large-amplitude collective motions of clusters
}

\author{
Tomohiro Yanao, ${ }^{\text {a) }}$ Wang Sang Koon, ${ }^{\text {b) }}$ and Jerrold E. Marsden ${ }^{\text {c) }}$ \\ Control and Dynamical Systems, MC 107-81, California Institute of Technology, Pasadena, \\ California 91125, USA
}

(Received 19 January 2009; accepted 20 February 2009; published online 13 April 2009)

\begin{abstract}
This paper uncovers novel and specific dynamical mechanisms that initiate large-amplitude collective motions in polyatomic molecules. These mechanisms are understood in terms of intramolecular energy transfer between modes and driving forces. Structural transition dynamics of a six-atom cluster between a symmetric and an elongated isomer is highlighted as an illustrative example of what is a general message. First, we introduce a general method of hyperspherical mode analysis to analyze the energy transfer among internal modes of polyatomic molecules. In this method, the $(3 n-6)$ internal modes of an $n$-atom molecule are classified generally into three coarse level gyration-radius modes, three fine level twisting modes, and $(3 n-12)$ fine level shearing modes. We show that a large amount of kinetic energy flows into the gyration-radius modes when the cluster undergoes structural transitions by changing its mass distribution. Based on this fact, we construct a reactive mode as a linear combination of the three gyration-radius modes. It is shown that before the reactive mode acquires a large amount of kinetic energy, activation or inactivation of the twisting modes, depending on the geometry of the isomer, plays crucial roles for the onset of a structural transition. Specifically, in a symmetric isomer with a spherical mass distribution, activation of specific twisting modes drives the structural transition into an elongated isomer by inducing a strong internal centrifugal force, which has the effect of elongating the mass distribution of the system. On the other hand, in an elongated isomer, inactivation of specific twisting modes initiates the structural transition into a symmetric isomer with lower potential energy by suppressing the elongation effect of the internal centrifugal force and making the effects of the potential force dominant. This driving mechanism for reactions as well as the present method of hyperspherical mode analysis should be widely applicable to molecular reactions in which a system changes its overall mass distribution in a significant way. (C) 2009 American Institute of Physics.
\end{abstract}

[DOI: $10.1063 / 1.3098141]$

\section{INTRODUCTION}

Molecular reactions are typically large-amplitude collective motions that involve a large number of degrees of freedom in a coherent manner. Understanding the mechanism for such collective motions is a significant challenge in chemical and biomolecular physics. The standard reaction rate theories, such as transition state theory ${ }^{1-6}$ and Rice-RamspergerKassel-Marcus (RRKM) theory, ${ }^{7-10}$ generally postulate that the internal energy of a molecule is quickly redistributed among the vibrational modes and the probability for a reaction coordinate (or a reactive mode) to acquire a sufficient amount of energy for reaction is determined statistically. Despite the great success of these standard theories, finding an appropriate reaction coordinate is generally not a trivial matter. Moreover, the statistical assumption of the reaction rate theories sometimes breaks down. ${ }^{6,10-14}$ Therefore, an important challenge remains; namely, to develop a general methodology for constructing a reaction coordinate and for under-

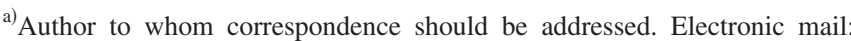
tyanao@cds.caltech.edu.

b) Electronic mail: koon@cds.caltech.edu.

c)Electronic mail: jmarsden@ caltech.edu.
}

standing the nonlinear dynamical processes that lead to large-amplitude collective motions. The purpose of this paper is to shed light on these fundamental issues of molecular reactions from the viewpoint of intramolecular energy transfer that is based on a novel method of mode analysis.

A standard method for mode analysis, namely, normal mode analysis, ${ }^{6,15}$ has been developed for a wide variety of systems including atomic liquids ${ }^{16,17}$ and biopolymers. ${ }^{18,19}$ While normal mode analysis is useful in describing smallamplitude vibrations of a system around an equilibrium (or an arbitrary reference) point in the configuration space, it may not be the most efficient way to study large-amplitude motions of molecules. This is because the normal mode concept is based on the local properties of the potential energy surface, while large-amplitude motions such as molecular reactions are not necessarily a local phenomenon on the potential energy surface. Rather, they represent a global movement in configuration space or, more accurately, in phase space. Therefore, a different method suitable for large-amplitude motions would be of complementary usefulness. Another controversial point of normal mode analysis as it applies to large-amplitude motions of molecules can be the problem of the separation of rotations and vibrations. A standard method 
is to use the so-called Eckart frame $e^{20,21}$ to approximately separate the rotational degrees of freedom from the vibrational ones. However, the Eckart frame is also based on the local geometry of the molecular configuration space, and this approximation may not be accurate enough in the study of large-amplitude collective motions.

This paper proposes a complementary method for mode analysis based on a concise expression for kinetic energy of an $n$-body system. While the potential energy surfaces are believed to govern the dynamics of molecules, the kinetic energy can also play an important role via the non-Euclidean mass matrix or metric tensor on the molecular internal space $^{22-25}$ and deserves closer attention. ${ }^{26-28}$ Moreover, while the potential energy surface changes from system to system, the expression of the kinetic energy is independent of the system once mass-weighted coordinates are used, indicating that the roles of kinetic energy are of rather universal nature. Therefore, the first motivation for our present study is to exploit a method of mode analysis in which the roles of the kinetic energy become transparent. For this purpose, the system of principal-axis hyperspherical coordinates $^{29-38}$ is remarkably suitable.

As was shown in Refs. 29-31, the use of gyration radii and quasivelocities associated with these coordinates allows the internal kinetic energy for an arbitrary $n$-atom molecule to be expressed in a very simple manner without any cross terms among the quasivelocities. Moreover, this kinetic energy expression can be further transformed into another concise one without the Coriolis coupling term between the rotational and the internal modes. All this can be done based on the framework of geometric mechanics ${ }^{39-43}$ and gauge theory. ${ }^{44-47}$ Inspired by such a concise expression of kinetic energy, this paper clarifies the physical meanings of the respective terms of kinetic energy and proposes a method of hyperspherical mode analysis, which classifies, for general systems, the $(3 n-6)$ internal modes of an $n$-atom system into three gyration-radius modes, three twisting modes, and $(3 n$ -12) shearing modes.

We apply the resulting hyperspherical mode analysis to the structural transition dynamics of a six-atom cluster $\left(\mathrm{Ar}_{6}\right.$-like cluster), which serves as an illustrative example of molecular reactions throughout this paper. This cluster undergoes structural transitions between two geometrically distinct isomers, one of which has a symmetric structure with spherical mass distribution and the other isomer has an elongated geometry. This cluster has been frequently studied from various perspectives such as potential energy topography, ${ }^{48,49}$ phase transitions, ${ }^{50,51}$ and nonlinear reaction dynamics. $^{52}$

Once the internal modes of the cluster are characterized using the hyperspherical modes, an important next step is to identify the predominant modes in structural transitions and construct an appropriate reactive mode and the corresponding reaction coordinate. Indeed, finding an appropriate reaction coordinate has been a longstanding subject in chemical physics. In the literature ${ }^{53-55}$ the steepest descent path connecting a saddle point and the minima on the potential energy surface has been frequently used as a reaction coordinate. Recent methods associated with the so-called transition paths ${ }^{56-61}$ propose another scheme to determine the reaction coordinate, the transition state, and the reaction rates based on an ensemble of reactive trajectories. Moreover, phase space approaches associated with cylindrical invariant manifolds ${ }^{62-66}$ provide a dynamical perspective on reaction coordinates and have been successfully applied to molecular reactions with a relatively small number of degrees of freedom in the computation of reaction rates. The latter two approaches, the transition-path and the phase space approach, are similar in that both highlight the distinction between reactive and nonreactive trajectories. This distinction seems to have a significant advantage in investigating the mechanisms of molecular reactions. Therefore, the basic strategy of the present study is to fully utilize an ensemble of reactive trajectories for identifying the predominant modes, for constructing a reactive mode/reaction coordinate, and for clarifying the driving mechanisms for structural transitions.

As a criterion to identify the most important modes in reactions, we postulate that the predominant modes are the modes that have a large amount of kinetic energy in the course of structural transition in the reactive trajectories. This criterion for predominant modes is based on the standard assumption of RRKM theory ${ }^{7-10}$ that molecular reactions take place when a critical degree of freedom (critical oscillator) acquires a sufficient amount of energy. In addition, modern phase space approaches ${ }^{62-66}$ establish this criterion rigorously, where reactive trajectories are characterized as the trajectories that have a sufficient amount of kinetic energy above a threshold in a reactive mode near a saddle point. Based on this criterion in terms of kinetic energy partitioning, we show that the gyration-radius modes are the predominant modes having a larger amount of kinetic energy in the course of structural transitions of clusters than the twisting and shearing modes. This result gives additional support for the idea of using molecular gyration radii as the building blocks for the reaction coordinate, as has been done recently in Ref. 28.

The paper, Ref. 28, is based on the use of the three gyration-radius modes as the coarse variables; ${ }^{67,68}$ that is, it identifies these as the "slow" variables, with the remaining variables being "fast." The present paper goes beyond this approach and uses energetic ideas to identify the specific mechanisms by which the fast modes induce transitions in the coarse modes.

To accomplish this, we linearly transform the three gyration-radius modes into a single reactive mode and two "bath" modes using principal component analysis. ${ }^{69,70}$ Correspondingly, the three gyration radii are transformed into a single reaction coordinate and two bath coordinates. The twisting and shearing modes are also regarded as bath modes at this stage.

After constructing the reactive mode using the gyrationradius modes, a next natural question regarding the mechanism for reaction is how the reactive mode acquires a large amount of kinetic energy from other modes prior to the onset of structural transitions inside potential wells. Recent experiments ${ }^{11-14}$ along with nonlinear dynamics studies ${ }^{71}$ indicate that certain characteristic pathways or steps of energy transfer may exist prior to the onset of reactions, which can 
cause a significant breakdown of the statistical assumptions in conventional reaction rate theories. ${ }^{6,10}$ In the present study, we investigate such characteristic energy transfer processes by making use of hyperspherical mode analysis. We then highlight the fact that the activation or inactivation of the twisting modes occurs, depending on the geometry of the isomer, before the reactive mode acquires a large amount of energy to start structural transitions.

A main goal of this paper is to understand the physical significance of the activation and inactivation of specific twisting modes that occur before the activation of the reactive mode in respective isomers. We solve this problem in terms of the kinematic mode coupling between the reactive mode and the twisting modes by investigating the equations of motion for the reaction coordinate, which is a linear combination of the gyration radii. As has been studied in Refs. 28,72 , and 73 , the equations of motion for the gyration radii reveal two different kinds of forces acting on the gyration radii, the potential force and a kinematic force called the internal centrifugal force. While the potential force has a general tendency to maintain the mass distribution of respective isomers, the internal centrifugal force has a persistent tendency to elongate the mass distribution of the system; that is, it is a symmetry breaking effect. Importantly, the magnitude of the elongation effect of the internal centrifugal force critically depends on the activity of the twisting modes. Based on this fact, the critical roles of the twisting modes for the onset of structural transitions in respective isomers is clarified. Specifically, in a symmetric isomer with a spherical mass distribution, activation of specific twisting modes drives the structural transition into an elongated isomer by inducing the strong internal centrifugal force. On the other hand, in an elongated isomer, inactivation of specific twisting modes initiates the structural transition into a symmetric isomer with lower potential energy by suppressing the elongation effect of the internal centrifugal force and making the effects of the potential force dominant.

As is discussed further in the conclusions at the end of the paper, we believe that the identification of these specific activation modes will ultimately lead to improved molecular control strategies.

This paper is organized as follows. In Sec. II, we introduce the definitions of hyperspherical modes in a general setting, and then clarify their physical meaning by taking an example of a six-atom cluster. In Sec. III, we show the predominance of the gyration-radius modes over all other hyperspherical modes in the structural transitions of the cluster from the viewpoint of energetics. We then construct a onedimensional reactive mode as a linear combination of the gyration-radius modes using the method of principal component analysis. All other hyperspherical modes are regarded as bath modes. In Sec. IV, we investigate the mechanism of energy transfer from the bath modes into the reactive mode prior to the onset of structural transitions. We then highlight that the activation or inactivation of the twisting modes, depending on the geometry of the isomer, plays critical roles in initiating structural transitions by inducing driving forces for large-amplitude collective motions. This paper concludes in Sec. V with some remarks on future directions of research.

\section{HYPERSPHERICAL MODES}

In this section, we introduce the definitions of hyperspherical modes for $n$-atom systems in a general setting based on a concise expression of the internal kinetic energy. Then we clarify their physical meaning by taking an example of a six-atom cluster, whose structural transition dynamics serves as an illustrative model of molecular reactions throughout this paper.

\section{A. The kinetic energy in principal-axis hyperspherical coordinates}

We summarize below the derivation of a concise expression of kinetic energy in principal-axis hyperspherical coordinates [see Eq. (9)]. The reader can refer to Ref. 28 for some additional details. Given a system of $n$ atoms, with masses $m_{i}(i=1, \ldots, n)$ and positions $\boldsymbol{r}_{i}=\left(r_{i x}, r_{i y}, r_{i z}\right)^{T}$, its overall translational degrees of freedom can be eliminated via the introduction of the $(n-1)$ mass-weighted Jacobi vectors,

$$
\boldsymbol{\rho}_{i}=\sqrt{\mu_{i}}\left(\frac{\sum_{k=1}^{i} m_{k} \boldsymbol{r}_{k}}{\sum_{k=1}^{i} m_{k}}-\boldsymbol{r}_{i+1}\right) \quad(i=1, \ldots, n-1)
$$

where $\mu_{i}$ are the reduced masses,

$$
\mu_{i}=\frac{m_{i+1} \Sigma_{k=1}^{i} m_{k}}{\sum_{k=1}^{i+1} m_{k}} \quad(i=1, \ldots, n-1) .
$$

Let $\mathbf{W} \equiv\left(\boldsymbol{\rho}_{1} \cdots \boldsymbol{\rho}_{n-1}\right)$ be a $3 \times(n-1)$-dimensional matrix whose columns are the $(n-1)$ Jacobi vectors of the system. According to the singular-value decomposition theorem, the matrix $\mathbf{W}$ can be decomposed into the product of three matrices as

$$
\mathbf{W}=\mathbf{R N U}^{T},
$$

where $\mathbf{R}$ is a three-dimensional orthogonal matrix whose three columns represent the instantaneous principal axes of the whole system. The matrix $\mathbf{N}$ is a $3 \times(n-1)$ "diagonal" matrix of the following structure:

$$
\mathbf{N}=\left(\begin{array}{cccccc}
a_{1} & 0 & 0 & 0 & \cdots & 0 \\
0 & a_{2} & 0 & 0 & \cdots & 0 \\
0 & 0 & a_{3} & 0 & \cdots & 0
\end{array}\right),
$$

where the singular values $a_{1}, a_{2}$, and $a_{3}$ are the gyration radii of the system. We caution that this definition of gyration radii does not coincide with the usual definition in the mechanics literature (see, for example Ref. 43). However, the three variables $a_{1}, a_{2}$, and $a_{3}$ in this paper still have the physical meaning of radii of gyration, being invariant under both external and kinematic (internal) rotations. ${ }^{30-38}$ In order to clarify the distinction between $a_{1}, a_{2}$, and $a_{3}$ in this paper and the usual gyration radii in mechanics, the former might better be called the hyperspherical gyration radii. But just for simplicity, we call $a_{1}, a_{2}$, and $a_{3}$ the gyration radii throughout this paper. Physically, the gyration radii represent the mass distribution of the system with respect to the three principal axes and are related to the three principal moments of inertia $M_{1} \leq M_{2}$ $\leq M_{3}$ as 


$$
M_{3}=a_{1}^{2}+a_{2}^{2}, \quad M_{2}=a_{1}^{2}+a_{3}^{2}, \quad M_{1}=a_{2}^{2}+a_{3}^{2} .
$$

That is,

$$
\begin{aligned}
& a_{1}^{2}=\frac{1}{2}\left(M_{2}+M_{3}-M_{1}\right), \quad a_{2}^{2}=\frac{1}{2}\left(M_{1}+M_{3}-M_{2}\right), \\
& a_{3}^{2}=\frac{1}{2}\left(M_{1}+M_{2}-M_{3}\right) .
\end{aligned}
$$

The matrix $\mathbf{U}$ in Eq. (3) is an $(n-1) \times(n-1)$ orthogonal matrix of the form $\mathbf{U} \equiv\left(\boldsymbol{u}_{1} \cdots \boldsymbol{u}_{n-1}\right)$, where the $(n-1)$-dimensional vectors $\left\{\boldsymbol{u}_{i}\right\}$ are orthogonal to each other and normalized. In the framework of hyperspherical coordinates, ${ }^{30}$ the matrix $\mathbf{U}$ can be parametrized by the $(3 n$ -9) hyperangles by restricting the matrix $\mathbf{U}$ to a subset of $O(n-1)$. Together with $a_{1}, a_{2}$, and $a_{3}$, these $(3 n-6)$ variables in the matrices $\mathbf{N}$ and $\mathbf{U}$ are the internal coordinates that characterize the shape of the system uniquely, while the principal-axis frame $\mathbf{R}$ specifies the orientation of the whole system. The use of the $(3 n-9)$ hyperangles generally makes the expression of kinetic energy highly involved. Instead, the use of $(3 n-9)$ quasivelocities makes the expression of kinetic energy concise as summarized below.

Based on the singular-value decomposition [Eq. (3)], the translation-reduced kinetic energy of the $n$-atom system can be generally expressed as

$$
K=\frac{1}{2} \sum_{i=1}^{n-1} \dot{\boldsymbol{\rho}}_{i} \cdot \dot{\boldsymbol{\rho}}_{i}=K_{\mathrm{rot}}+K_{\mathrm{int}}
$$

Here, $K_{\text {rot }}$ is the rotational kinetic energy given by

$$
K_{\text {rot }}=\frac{1}{2} \boldsymbol{L}^{T} \mathbf{M}^{-1} \boldsymbol{L},
$$

where $\boldsymbol{L}$ is a three-dimensional vector of the angular momentum with respect to the principal-axis frame and $\mathbf{M}$ is its corresponding $3 \times 3$ moment of inertia tensor. $K_{\text {int }}$ is the internal kinetic energy and can be given explicitly as

$$
\begin{aligned}
K_{\mathrm{int}}= & \frac{1}{2}\left(\dot{a}_{1}^{2}+\dot{a}_{2}^{2}+\dot{a}_{3}^{2}\right)+\frac{\left(a_{1}^{2}-a_{2}^{2}\right)^{2}}{2\left(a_{1}^{2}+a_{2}^{2}\right)} \omega_{12}^{2}+\frac{\left(a_{2}^{2}-a_{3}^{2}\right)^{2}}{2\left(a_{2}^{2}+a_{3}^{2}\right)} \omega_{23}^{2} \\
& +\frac{\left(a_{3}^{2}-a_{1}^{2}\right)^{2}}{2\left(a_{3}^{2}+a_{1}^{2}\right)} \omega_{31}^{2}+\frac{1}{2} a_{1}^{2} \sum_{k=4}^{n-1} \gamma_{1 k}^{2}+\frac{1}{2} a_{2}^{2} \sum_{k=4}^{n-1} \gamma_{2 k}^{2} \\
& +\frac{1}{2} a_{3}^{2} \sum_{k=4}^{n-1} \gamma_{3 k}^{2},
\end{aligned}
$$

where $\left\{\omega_{i j}\right\}$ and $\left\{\gamma_{i k}\right\}$ are the quasivelocities defined by

$$
\begin{aligned}
& \dot{\boldsymbol{u}}_{i} \cdot \boldsymbol{u}_{j}=-\boldsymbol{u}_{i} \cdot \dot{\boldsymbol{u}}_{j} \equiv \omega_{i j} \quad(i, j=1,2,3, i \neq j), \\
& \dot{\boldsymbol{u}}_{i} \cdot \boldsymbol{u}_{k}=-\boldsymbol{u}_{i} \cdot \dot{\boldsymbol{u}}_{k} \equiv \gamma_{i k} \quad(i=1,2,3, k=4, \ldots, n-1) .
\end{aligned}
$$

The vectors $\left\{\boldsymbol{u}_{i}\right\}$ are the $(n-1)$-dimensional vectors defined in the matrix $\mathbf{U}$ in Eq. (3). The quasivelocities $\left\{\omega_{i j}\right\}$ are antisymmetric with respect to the exchange of the suffixes as $\omega_{i j}=-\omega_{j i}$. Both $\left\{\omega_{i j}\right\}$ and $\left\{\gamma_{i k}\right\}$ are the parts of the components of the $(n-1) \times(n-1)$ antisymmetric matrix $\mathbf{U}^{T} \dot{\mathbf{U}}$, which is essentially an internal angular velocity associated with the shape changes in the system. We clarify the physical meanings of these quasivelocities in the following two sub- sections. Note that the expression (9) is valid for general $n$-atom $(n \geq 5)$ systems. If $n \leq 4$, the quasivelocities $\left\{\gamma_{i k}\right\}$ do not exist, and all the terms involving $\left\{\gamma_{i k}\right\}$ disappear from Eq. (9) (see Ref. 72 for three- and four-atom systems). Hereafter, we assume the general case of $n \geq 5$ unless otherwise noted.

An advantage of the expression (7) is that there is no Coriolis coupling term, and the partitioning of $K$ into $K_{\text {rot }}$ and $K_{\text {int }}$ is independent of the choice of body frame. [It should be noted, however, that the terms on the right-hand side of Eq. (9) have physical meanings specifically with respect to the principal-axis frame as will be shown in the following subsections.] When the total angular momentum of the system is zero, $\boldsymbol{L}=\mathbf{0}$, the rotational kinetic energy $K_{\text {rot }}$ disappears and $K_{\text {int }}$ is essentially the total kinetic energy of the system.

The internal kinetic energy equation (9) is remarkably simple in the sense that there is no cross term among the velocities of gyration radii $\left\{\dot{a}_{i}\right\}$ and the quasivelocities $\left\{\omega_{i j}\right\}$ and $\left\{\gamma_{i k}\right\}$. Moreover, the number of terms on the right-hand side of Eq. (9) is $(3 n-6)$ in total, which is exactly the same as the number of internal degrees of freedom of the $n$-atom system. Therefore, it is tempting to consider the terms of Eq. (9) as the kinetic energies of respective $(3 n-6)$ internal degrees of freedom. The first three terms proportional to $\dot{a}_{1}^{2}, \dot{a}_{2}^{2}$, and $\dot{a}_{3}^{2}$ are clearly the kinetic energies of the three gyration radii. But for the rest of the terms involving $\left\{\omega_{i j}\right\}$ and $\left\{\gamma_{i k}\right\}$, it is not as simple because one cannot attribute them to any coordinates since these quasivelocities are not derivatives of coordinates. Instead, these kinetic energies are the energies of respective internal modes characterized by the quasivelocities $\left\{\omega_{i j}\right\}$ and $\left\{\gamma_{i k}\right\}$ themselves. In the following two subsections, we clarify the physical meanings of these internal modes that correspond to the quasivelocities $\left\{\omega_{i j}\right\}$ and $\left\{\gamma_{i k}\right\}$. We then propose to use the concise expression of the kinetic energy equation (9) as a fundamental tool to investigate the intramolecular energy transfer in the dynamics of $n$-atom systems as will be demonstrated in the later sections.

\section{B. Definitions of hyperspherical modes}

We introduce below the definitions of hyperspherical modes, which are inherent in the concise expression for the internal kinetic energy equation (9). While the variables $\left\{\dot{a}_{i}\right\}$, $\left\{\omega_{i j}\right\}$, and $\left\{\gamma_{i k}\right\}$ in Eq. (9) are the components of a given velocity vector in the tangent space of the configuration space, the hyperspherical modes themselves are defined as the basis vectors conjugate to these components.

Suppose that $\dot{\mathbf{W}}$ is a velocity vector of the system that satisfies the conditions of zero total angular momentum. Note that this $3 \times(n-1)$ matrix $\dot{\mathbf{W}}$ can be regarded as a vector in the tangent space of the (3n-3)-dimensional translation-reduced configuration space by aligning all the columns of $\dot{\mathbf{W}}$ to a single column. As long as the vector $\dot{\mathbf{W}}$ satisfies the conditions of zero total angular momentum, $\mathbf{W}$ can be expanded into $(3 n-6)$ terms as 


$$
\dot{\mathbf{W}}=\sum_{i=1}^{3} \dot{a}_{i} \mathbf{V}_{a_{i}}+\sum_{i j=12,23,31} \omega_{i j} \mathbf{V}_{\omega_{i j}}+\sum_{i=1}^{3} \sum_{k=4}^{n-1} \gamma_{i k} \mathbf{v}_{\gamma_{i k}},
$$

where $\left\{\mathbf{V}_{a_{i}}\right\},\left\{\mathbf{V}_{\omega_{i j}}\right\}$, and $\left\{\mathbf{V}_{\gamma_{i k}}\right\}$ are $3 \times(n-1)$ matrices, which constitute the $(3 n-6)$ basis vectors of this expansion. Each of these basis vectors corresponds to a hyperspherical mode. In what follows, we deduce the expressions for these basis vectors.

We begin with the following expression for $\dot{\mathbf{W}}$ :

$$
\dot{\mathbf{W}}=\mathbf{R} \dot{\mathbf{N}} \mathbf{U}^{T}+\mathbf{R}\left(\boldsymbol{\Omega N}+\mathbf{N} \boldsymbol{\omega}^{T}\right) \mathbf{U}^{T},
$$

which is obtained from the time derivative of Eq. (3). In Eq. (13), the matrix $\boldsymbol{\Omega} \equiv \mathbf{R}^{T} \mathbf{R}$ is a $3 \times 3$ antisymmetric matrix corresponding to the angular velocity of the principal-axis frame with respect to the principal-axis frame itself. The matrix $\boldsymbol{\omega} \equiv \mathbf{U}^{T} \dot{\mathbf{U}}$ is an $(n-1) \times(n-1)$ antisymmetric matrix that can be expanded as

$$
\boldsymbol{\omega}=\sum_{i j=12,23,31} \omega_{i j} \mathbf{X}_{i j}+\sum_{i=1}^{3} \sum_{k=4}^{n-1} \gamma_{i k} \mathbf{X}_{i k}+\sum_{k=4}^{n-2} \sum_{l=k+1}^{n-1} \gamma_{k l} \mathbf{x}_{k l},
$$

where $\mathbf{X}_{i j}$ are defined as the $(n-1) \times(n-1)$-dimensional antisymmetric matrix whose $i j$ component is $-1, j i$ component is +1 , and all other components are zero. The matrices $\mathbf{X}_{i k}$ and $\mathbf{X}_{k l}$ have the similar structure, whose $i k$ or $k l$ component is -1 and the $k i$ or $l k$ component is +1 . In the first two terms on the right-hand side of Eq. (14), $\left\{\omega_{i j}\right\}$ and $\left\{\gamma_{i k}\right\}$ are the quasivelocities introduced in Eqs. (10) and (11). In the third term of Eq. (14), we have introduced additional quasivelocities defined by

$$
\dot{\boldsymbol{u}}_{k} \cdot \boldsymbol{u}_{l}=-\boldsymbol{u}_{k} \cdot \dot{\boldsymbol{u}}_{l} \equiv \gamma_{k l} \quad(k, l=4, \ldots, n-1, k<l) .
$$

The quasivelocities in Eq. (15) vanish after the matrix multiplication $\mathbf{N} \boldsymbol{\omega}^{T}$ in Eq. (13).

Our strategy here is to express Eq. (13) in the form of Eq. (12) and compare these two equations to deduce the basis vectors $\left\{\mathbf{V}_{a_{i}}\right\},\left\{\mathbf{V}_{\omega_{i j}}\right\}$, and $\left\{\mathbf{V}_{\gamma_{i k}}\right\}$. So far, Eq. (13) is more general than Eq. (12) because Eq. (13) includes the situation that $\dot{\mathbf{W}}$ gives nonzero total angular momentum. Therefore, we need to assign the conditions of vanishing total angular momentum to Eq. (13). If the velocity vector $\dot{\mathbf{W}}$ in Eq. (13) satisfies the conditions of vanishing total angular momentum, one can eliminate the angular velocity of the principalaxis frame $\boldsymbol{\Omega}$ by expressing it in terms of the internal variables as is known from the gauge theory. ${ }^{47}$ This can be done as follows.

The total angular momentum of the system (with respect to the space-fixed frame) for a given velocity vector $\dot{\mathbf{W}}$ can be expressed in the form of a $3 \times 3$ antisymmetric matrix as

$$
\mathbf{L}_{s}=\dot{\mathbf{W}} \mathbf{W}^{T}-\mathbf{W} \dot{\mathbf{W}}^{T} .
$$

By inserting Eq. (13) into Eq. (16), we obtain

$$
\mathbf{L}_{s}=\mathbf{R}\left(\mathbf{\Omega} \mathbf{N} \mathbf{N}^{T}+\mathbf{N} \boldsymbol{\omega}^{T} \mathbf{N}^{T}-\mathbf{N} \mathbf{N}^{T} \boldsymbol{\Omega}^{T}-\mathbf{N} \boldsymbol{\omega} \mathbf{N}^{T}\right) \mathbf{R}^{T} .
$$

Using this equation, the condition of vanishing total angular momentum, $\mathbf{L}_{s}=\mathbf{0}$, can be solved for $\boldsymbol{\Omega}$ to give

$$
\begin{aligned}
\boldsymbol{\Omega} & =\left(\begin{array}{ccc}
0 & -\frac{2 a_{1} a_{2}}{a_{1}^{2}+a_{2}^{2}} \omega_{12} & \frac{2 a_{3} a_{1}}{a_{3}^{2}+a_{1}^{2}} \omega_{31} \\
\frac{2 a_{1} a_{2}}{a_{1}^{2}+a_{2}^{2}} \omega_{12} & 0 & -\frac{2 a_{2} a_{3}}{a_{2}^{2}+a_{3}^{2}} \omega_{23} \\
-\frac{2 a_{3} a_{1}}{a_{3}^{2}+a_{1}^{2}} \omega_{31} & \frac{2 a_{2} a_{3}}{a_{2}^{2}+a_{3}^{2}} \omega_{23} & 0
\end{array}\right) \\
& \equiv \mathbf{A}_{12} \omega_{12}+\mathbf{A}_{23} \omega_{23}+\mathbf{A}_{31} \omega_{31},
\end{aligned}
$$

where, in the final equality, $\mathbf{A}_{i j}(i j=12,23,31)$ are the 3 $\times 3$ antisymmetric matrices whose $i j$ components are $-2 a_{i} a_{j} /\left(a_{i}^{2}+a_{j}^{2}\right)$ and $j i$ components are $+2 a_{i} a_{j} /\left(a_{i}^{2}+a_{j}^{2}\right)$ and all other components are zero. Physically, Eq. (18) is the angular velocity of the principal-axis frame that compensates for the angular momentum induced by the quasivelocities $\omega_{12}, \omega_{23}$, and $\omega_{31}$ to keep the total angular momentum zero. After inserting Eq. (18) into Eq. (13) and using Eq. (14) for $\boldsymbol{\omega}$, we obtain a general expression for the velocity vector $\mathbf{W}$ under the conditions of vanishing total angular momentum as

$$
\begin{aligned}
\dot{\mathbf{W}}= & \sum_{i=1}^{3} \dot{a}_{i} \mathbf{R} \frac{\partial \mathbf{N}}{\partial a_{i}} \mathbf{U}^{T}+\sum_{i j=12,23,31} \omega_{i j} \mathbf{R}\left(\mathbf{A}_{i j} \mathbf{N}+\mathbf{N} \mathbf{X}_{i j}^{T}\right) \mathbf{U}^{T} \\
& +\sum_{i=1}^{3} \sum_{k=4}^{n-1} \gamma_{i k} \mathbf{R} \mathbf{N} \mathbf{X}_{i k}^{T} \mathbf{U}^{T} .
\end{aligned}
$$

By comparing Eq. (19) with Eq. (12), we see that the three gyration-radius modes can be defined as

$$
\mathbf{V}_{a_{i}} \equiv \mathbf{R} \frac{\partial \mathbf{N}}{\partial a_{i}} \mathbf{U}^{T} \quad(i=1,2,3) .
$$

Similarly, the first three hyperangular modes corresponding to $\omega_{12}, \omega_{23}$, and $\omega_{31}$ can be defined as

$$
\mathbf{V}_{\omega_{i j}} \equiv \mathbf{R}\left(\mathbf{A}_{i j} \mathbf{N}+\mathbf{N} \mathbf{X}_{i j}^{T}\right) \mathbf{U}^{T} \quad(i j=12,23,31),
$$

and other hyperangular modes corresponding to $\gamma_{i k}(i$ $=1,2,3, k=4, \ldots, n-1)$ can be defined as

$$
\mathbf{V}_{\gamma_{i k}} \equiv \mathbf{R} \mathbf{N} \mathbf{X}_{i k}^{T} \mathbf{U}^{T} \quad(i=1,2,3, k=4, \ldots, n-1) .
$$

Thus, Eqs. (20)-(22) give the definitions of the $(3 n-6)$ internal modes. Note that the hyperspherical modes in Eqs. (20)-(22) are now expressed solely by the quantities that are obtained through the singular value decomposition in Eq. (3). Therefore, one can immediately compute all the hyperspherical modes through Eqs. (20)-(22) once the singular value decomposition equation (3) is obtained.

It can be shown that the hyperangular modes defined above are orthogonal to each other; in fact, we have

$$
\operatorname{Tr}\left[\mathbf{V}_{\alpha}^{T} \mathbf{V}_{\beta}\right]=0 \quad(\text { for } \alpha \neq \beta),
$$

where $\alpha$ and $\beta$ represent any of $\left\{a_{i}\right\},\left\{\omega_{i j}\right\}$, and $\left\{\gamma_{i k}\right\}$. These orthogonal properties indicate that the hyperspherical modes form a set of orthogonal basis vectors that span the tangent space of the configuration space under the conditions of zero total angular momentum, which is a property similar to ordinary normal modes. ${ }^{15}$ Furthermore, the hyperspherical modes are normalized to the coefficients of the kinetic energy in Eq. (9), 


$$
\begin{aligned}
& \operatorname{Tr}\left[\mathbf{V}_{a_{i}}^{T} \mathbf{V}_{a_{i}}\right]=1 \quad(i=1,2,3) \\
& \operatorname{Tr}\left[\mathbf{V}_{\omega_{i j}}^{T} \mathbf{V}_{\omega_{i j}}\right]=\left(a_{i}^{2}-a_{j}^{2}\right)^{2} /\left(a_{i}^{2}+a_{j}^{2}\right) \quad(i j=12,23,31), \\
& \operatorname{Tr}\left[\mathbf{V}_{\gamma_{i k}}^{T} \mathbf{V}_{\gamma_{i k}}\right]=a_{i}^{2} \quad(i=1,2,3, k=4, \ldots, n-1) .
\end{aligned}
$$

Note that the kinetic energy of the system given in Eq. (7) can also be expressed as $2 K=\operatorname{Tr}\left[\dot{\mathbf{W}}^{T} \mathbf{\mathbf { W }}\right]$. By inserting Eq. (12) into this kinetic energy expression, we can see that the orthonormal conditions, Eqs. (23)-(26), are vital to the concise expression of kinetic energy in Eq. (9) without any cross terms. Note finally that the hyperspherical modes are determined only by the quantities associated with kinetic energy, i.e., the atomic masses and the shape of the system, and are totally independent of the potential function of the system. In Sec. II C, we will clarify the physical meaning of each hyperspherical mode by taking an illustrative example of a sixatom cluster.

\section{Representation of hyperspherical modes for an illustrative model system}

In this subsection, we clarify the physical meanings of the hyperspherical modes defined in Eqs. (20)-(22) based on an illustrative model of a six-atom cluster. Since the structural transition dynamics of this cluster is of great interest in later sections, we first summarize this model system briefly.

Our illustrative model is a cluster composed of six identical atoms that mutually interact through a pairwise Morse potential. This cluster, called the $\mathrm{M}_{6}$ cluster, and similar Lennard-Jones clusters have been studied extensively from various perspectives such as potential energy topography, ${ }^{48,49}$ phase transitions, ${ }^{50,51}$ and nonlinear dynamics. ${ }^{52}$ The dimensionless Hamiltonian of the system is given by

$$
\frac{\mathcal{H}}{\varepsilon}=\frac{1}{2} \sum_{i=1}^{6}\left(\dot{\boldsymbol{r}}_{i} \cdot \dot{\boldsymbol{r}}_{i}\right)+\sum_{i<j}\left[e^{-2\left(d_{i j}-d_{0}\right)}-2 e^{-\left(d_{i j}-d_{0}\right)}\right],
$$

where $\boldsymbol{r}_{i}(i=1, \ldots, 6)$ is the three-dimensional position vector of the atom $i$. The dot over $\boldsymbol{r}_{i}$ represents the time derivative. All the masses of the atoms can be regarded as unity in the dimensionless Hamiltonian. The parameter $\varepsilon$ represents the depth of the Morse potential and $d_{i j}$ is the interparticle distance between atom $i$ and atom $j$. The parameter $d_{0}$, which corresponds to the equilibrium distance of the pairwise Morse potential, is set to $d_{0}=6.0$. This provides a potential topography similar to that of the Lennard-Jones potential. ${ }^{49}$ Therefore, this system can be regarded as a model of the $\mathrm{Ar}_{6}$ cluster.

The isomerization scheme of the $\mathrm{M}_{6}$ cluster is shown in Fig. 1. This cluster has two geometrically distinct isomers: One is the regular octahedron (OCT) and the other is the capped trigonal bipyramid (CTBP). ${ }^{48,49,52}$ The potential energy minimum of the OCT isomer is $V=-12.49 \varepsilon$, and that of the CTBP isomer is $V=-12.13 \varepsilon$. These two isomers are connected through a saddle point whose potential energy is $V=$ $-11.83 \varepsilon$. In Fig. 1, the potential energy topography along the steepest descent path is shown. The OCT isomer has a highly

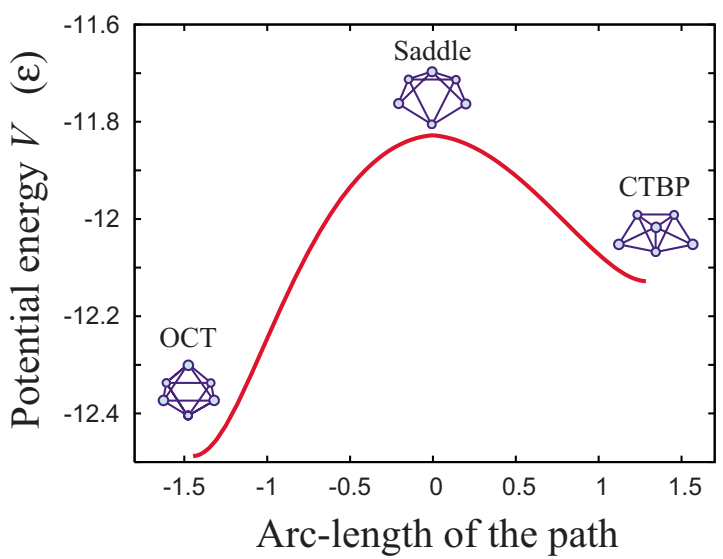

FIG. 1. (Color online) Isomerization scheme of the six-atom Morse cluster. The cluster has two geometrically distinct isomers, OCT and CTBP. The potential energy curve along the steepest descent path connecting the saddle point and the two potential minima is shown. The horizontal axis measures the arc length of the path, which quantifies the variation of the three gyration radii along the path. The potential energy of the OCT isomer at the minimum point is $V=-12.49 \varepsilon$, while that of the CTBP isomer is $V=-12.13 \varepsilon$. These minima are connected through a saddle point whose potential energy is $V=-11.83 \varepsilon$.

symmetric structure with a deep potential well, while the CTBP isomer has an elongated (collapsed) structure with a shallow potential well. Since the mass distribution of each isomer will be important in the later sections, we here note the following: The OCT isomer has a spherical mass distribution and the values of the gyration radii are $\left(a_{1}, a_{2}, a_{3}\right)$ $=(5.97,5.97,5.97)$ at the minimum of the potential energy. The CTBP isomer has an elongated mass distribution and the values of gyration radii are $\left(a_{1}, a_{2}, a_{3}\right)=(8.19,5.89,4.27)$ at the local minimum of potential energy. The horizontal axis of Fig. 1 measures the variation of the three gyration radii of the cluster along the path.

Throughout this paper, a microcanonical, constant energy simulation is employed. Since the main interest of this study is in the internal dynamics of polyatomic systems, the total angular momentum of the system is set to be zero throughout the paper. If the total energy of the cluster is higher than that of the saddle point, the isomerization reactions between the two isomers are energetically possible. In this paper, all the simulations are done at the total energy level of $E=-11.0 \varepsilon$. At this energy level, the cluster tends to stay in the two isomers for roughly the same amount of time in long-time simulations. ${ }^{28}$ In what follows, our numerical results are presented in absolute units.

We now investigate the physical meanings of the hyperspherical modes defined in Eqs. (20)-(22) based on the $\mathrm{M}_{6}$ cluster. For $n=6$, there are $(3 n-6)=12$ hyperspherical modes. Figure 2 shows the three-dimensional representation of these modes around the OCT [Figs. 2(a)-2(1)] and the CTBP [Figs. 2(n)-2(y)] isomers. In these figures, the cluster is aligned so that the first, second, and third principal axes are parallel to the axes indicated in $(\mathrm{m})$ for OCT and in $(\mathrm{z})$ for CTBP. Since the three gyration radii happen to be degenerate (equal) at the minimum of the potential energy in the OCT isomer, the corresponding principal axes are not uniquely determined. Therefore, for the sake of visualization, 
(a)

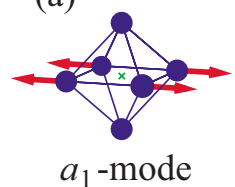

(d)

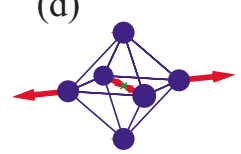

$\omega_{12}$-mode (b)

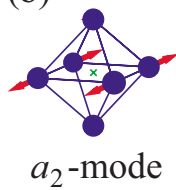

(e)

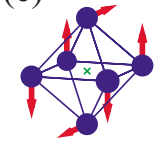

$\omega_{23}$-mode

(g)

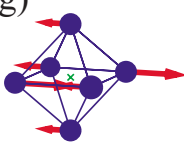

$\gamma_{14}$-mode

(i)

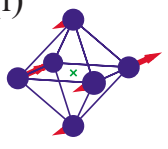

$\gamma_{24}$-mode

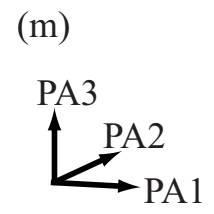

(k)

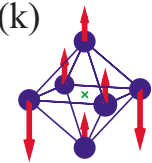

$\gamma_{34}$-mode

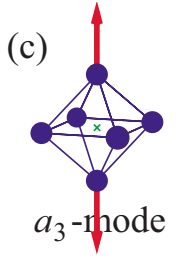

(f)

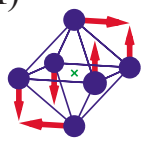

$\omega_{31}$-mode

(h)

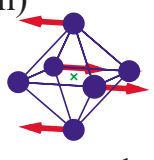

$\gamma_{15}$-mode

(j)

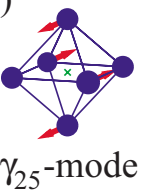

(1)

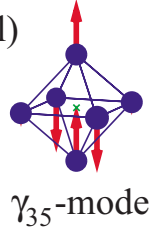

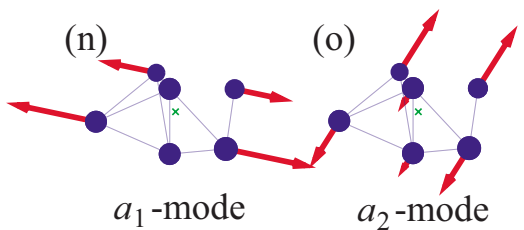
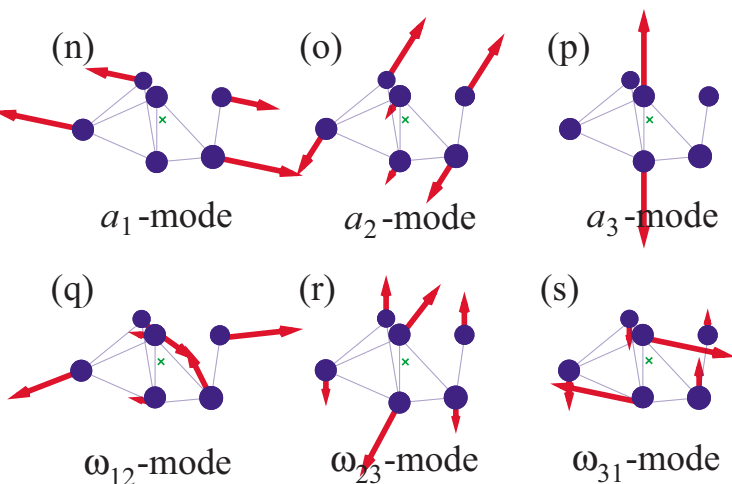

(S)

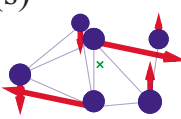

$\omega_{31}$-mode

(t)

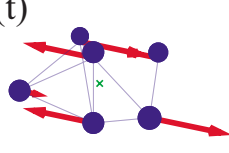

$\gamma_{14}$-mode

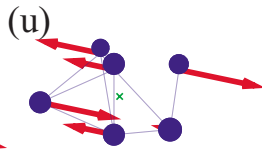

(v)
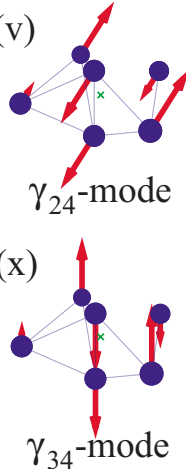

(z)

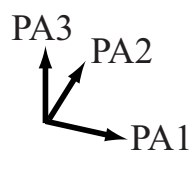

(w)

$\gamma_{15}$-mode
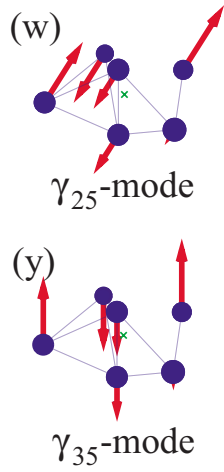

FIG. 2. (Color online) The 12 hyperspherical modes of the six-atom cluster, $\mathrm{M}_{6}$, around the OCT [(a)-(1)] and the CTBP isomers [(n)-(y)]. The cluster is aligned so that the first, second, and third principal axes (PA1, PA2, and PA3, respectively) are parallel to the axes indicated in (m) for OCT and in (z) for CTBP. (The OCT isomer shown here is slightly deformed from the potential energy minimum. See the text for detail.) The three gyration-radius modes, $a_{1}{ }^{-}$, $a_{2^{-}}$, and $a_{3}$-modes, shown in (a)-(c) and (n)-(p) for the two isomers, respectively, are related to the elongation and the contraction of the systems along the three principal axes. For the three twisting modes, shown in (d)-(f) and (q)-(s), the arrows in each $\omega_{i j}$-mode are perpendicular to the $k$ th $(k \neq i, j)$ principal axis; one part of the system tends to rotate around the $k$ th principal axis in one direction, and the other part of the system tends to rotate in the opposite direction, giving rise to a twist around the $k$ th principal axis. The remaining six modes associated with $\gamma_{i k}(i=1,2,3, k=4,5)$, shown in (g)-(l) and (t) $-(\mathrm{y})$, are the shearing modes; while all the atoms in each $\gamma_{i k}$-mode tend to move parallel to the $i$ th principal axis, some move in the positive direction and others move in the negative direction, giving rise to a shear in the system.

we have slightly deformed the OCT structure in the direction of the saddle point along the steepest descent path in Figs. 2(a)-2(1). Note, however, that the hyperspherical modes are well defined almost everywhere in the configuration space except for the points where two or three of the gyration radii are exactly the same, i.e., the singular values in Eq. (4) are degenerate.

The arrows in Fig. 2 represent the vectors of the hyperspherical modes defined in Eqs. (20)-(22). Since the vectors $\mathbf{V}_{a_{i}}, \mathbf{V}_{\omega_{i j}}$, and $\mathbf{V}_{\gamma_{i k}}$ in these equations are expressed in terms of Jacobi vectors, we converted Eqs. (20)-(22) into the corresponding expressions in Cartesian coordinates by using the inversion of Eq. (1) in obtaining the three-dimensional representations in Fig. 2. It is important to note that, in each picture of Fig. 2, the motion characterized by the arrows that are inverted all at the same time belongs to the same hyperspherical mode as the one shown in the figure. This is because the vectors $\mathbf{V}_{\alpha}$ and $-\mathbf{V}_{\alpha}$, where $\alpha$ represents any of $\left\{a_{i}\right\},\left\{\omega_{i j}\right\}$, and $\left\{\gamma_{i k}\right\}$, span the same tangent space and differ only in their signs. In addition, we need only one of either $\omega_{i j}$-mode or $\omega_{j i}$-mode as a basis because $\mathbf{V}_{\omega_{i j}}=-\mathbf{V}_{\omega_{j i}}$ holds by virtue of the antisymmetric property of $\mathbf{A}_{i j}$ and $\mathbf{X}_{i j}$ in Eq. (21).

Figures 2(a)-2(c) and 2(n)-2(p) show the $a_{1^{-}}, a_{2^{-}}$, and $a_{3}$-modes, which are the gyration-radius modes defined in Eq. (20) for the two isomers. It is clearly seen that the gyration-radius modes are related to inflation and contraction of the system along the three principal axes, respectively. Therefore, combinations of these gyration-radius modes characterize the change in the overall mass distribution of the system with respect to the three principal axes. The other three modes, $\omega_{12^{-}}, \omega_{23^{-}}$, and $\omega_{31^{-}}$-modes defined by Eq. (21) are shown in Fig. 2(d)-2(f) and 2(q)-2(s) for the respective isomers. As can be seen from these figures, all the arrows in the $\omega_{12^{-}}, \omega_{23^{-}}$, and $\omega_{31^{-}}$modes are perpendicular to the third, first, and second principal axes, respectively. Furthermore, in each of these $\omega_{i j}$-modes, one part of the system tends to rotate around the $k$ th $(k \neq i, j)$ principal axis in one direction, and the other part of the system tends to rotate in the opposite direction, giving rise to some twist around the $k$ th 
$(k \neq i, j)$ principal axis. Thus we call the $\omega_{12^{-}}, \omega_{23^{-}}$, and $\omega_{31}$-modes the twisting modes. Note that, since these modes are internal modes, they do not induce any total linear nor angular momentum due to the condition introduced in Eq. (18). The remaining six modes associated with $\gamma_{i k}(i$ $=1,2,3, k=4,5)$ are shown in Figs. $2(\mathrm{~g})-2(1)$ and $2(\mathrm{t})-2(\mathrm{y})$. In each of these $\gamma_{i k}$-modes, some atoms tend to move in one of the two directions parallel to the $i$ th principal axis, and the remaining atoms tend to move in the opposite direction, giving rise to a shear in the system. Thus, we call these $\gamma_{i k}$-modes the shearing modes.

In principle, one can determine the hyperspherical modes for arbitrary configurations of an $n$-atom system in the same way as above as long as the singular value decomposition equation (3) is well defined. In the case of a general $n$-atom system ( $n \geq 4)$, the numbers of the gyration-radius modes, twisting modes, and shearing modes are 3,3 , and $(3 n-12)$, respectively. Thus, only the number of shearing modes $\left(\gamma_{i k}\right.$-modes) increases when the number of atoms $n$ increases. If $n=4$, there are no shearing modes, and we have only three gyration-radius modes and three twisting modes. If $n=3$, we have only two gyration-radius modes and one twisting mode.

We note here that the $\gamma_{i k}$-modes have an arbitrariness with respect to the exchange of the suffixes $k$. For example, the $\gamma_{14}$-mode shown in Fig. 2 could also be labeled as the $\gamma_{15}$-mode and vice versa. This is due to the arbitrariness of the singular value decomposition of the matrix $\mathbf{W}$ [Eq. (3)] with respect to the ordering of the last $(n-4)$ columns $\boldsymbol{u}_{k}$ $(k=4, \ldots, n-1)$ of the matrix $\mathbf{U}$. In fact, the $\gamma_{i k}$-modes have a larger class of arbitrariness associated with the orthogonal transformations of $\mathbf{U}$ that act only on the last $(n-4)$ columns $\boldsymbol{u}_{k}(k=4, \ldots, n-1)$ without affecting the first three columns $\boldsymbol{u}_{k}(k=1,2,3)$. This arbitrariness in the $\gamma_{i k}$-modes leads to arbitrariness in the definitions of kinetic energies in the shearing modes: In the current framework, while the sums of kinetic energies in the shearing modes (with respect to the second subscript $k),\left(a_{i}^{2} / 2\right) \sum_{k=4}^{n-1} \gamma_{i k}^{2}$, which appear in Eq. (9), are uniquely determined for respective $i(=1,2,3)$, there is arbitrariness in the decomposition of each of these sums into the kinetic energies in the respective shearing modes, $K_{\gamma_{i k}}$ $\equiv\left(a_{i}^{2} / 2\right) \gamma_{i k}^{2}$, where $k=4, \ldots, n-1$. For example, in our sixatom cluster $(n=6)$, the sum of the kinetic energies in the $\gamma_{14}$-mode and $\gamma_{15}$-mode, $K_{\gamma_{14}}+K_{\gamma_{15}}$, is always unique and exact, whereas each of $K_{\gamma_{14}}$ and $K_{\gamma_{15}}$ is determined arbitrarily. This arbitrariness in the kinetic energies in the shearing modes should be noted in the following sections when we discuss the kinetic-energy partitioning among the internal modes in Figs. 4, 7, and 8. In order to resolve such arbitrariness in the shearing modes, one needs to introduce extra rules so that the columns $\boldsymbol{u}_{k}(k=4, \ldots, n-1)$ of $\mathbf{U}$ are uniquely determined. However, it is important that no matter how such rules are introduced, the resulting $\gamma_{i k}$-modes always have the physical meaning of the shearing modes along the $i$ th principal axis as is shown in Figs. 2(g)-2(1) and 2(t)$2(\mathrm{y})$. Thus, as far as the present study is concerned, we do not find significant physical motivation in resolving the arbitrariness in the shearing modes, and we do not deal with this arbitrariness here.
As mentioned earlier, an advantage of the hyperspherical modes is that the kinetic energy of each mode is exactly defined by the corresponding term of Eq. (9) (except for the arbitrariness in the shearing modes as mentioned above). Therefore one can scrutinize the flow of kinetic energy among the hyperspherical modes in conformational dynamics of arbitrary molecular systems. Such information on the kinetic energy flow can in turn provide crucial information for understanding the mechanism for large-amplitude collective motions. In the following sections, we demonstrate this procedure systematically by studying the structural transition dynamics of the $\mathrm{M}_{6}$ cluster.

\section{CONSTRUCTION OF A REACTIVE MODE AND A REACTION COORDINATE}

The purpose of this section is to construct a reactive mode and a corresponding reaction coordinate that plays a predominant role in the structural transitions of the $\mathrm{M}_{6}$ cluster. By applying the framework of the hyperspherical modes introduced in Sec. II, we first show that the gyration-radius modes are the most predominant modes having a large amount of kinetic energy in the course of structural transitions. Based on this fact, we transform the three gyrationradius modes into a single reactive mode and two bath modes by making use of principal component analysis. All other hyperspherical modes, the twisting modes, and the shearing modes are also regarded as bath modes. This classification of the internal modes serves as a basis for the study of the driving mechanisms for structural transitions in Sec. IV.

\section{A. Predominant hyperspherical modes in structural transition}

In this subsection, we extract the predominant modes in the structural transition of the $\mathrm{M}_{6}$ cluster from the 12 hyperspherical modes shown in Fig. 2. Our criterion for the predominant modes is that they must be highly active having a large amount of kinetic energy during the course of structural transitions. This criterion for the predominant modes is based on the standard picture for reactions in the RRKM theory, ${ }^{7-10}$ where it is generally postulated that molecular reactions take place when a certain critical degree of freedom (or a critical oscillator) acquires a sufficient amount of energy. The legitimacy of this picture is further confirmed by the modern phase space approaches, ${ }^{62-66}$ where it is clearly shown that a reactive mode possesses a sufficient amount of kinetic energy above a threshold near a saddle point in an ensemble of reactive trajectories. Thus, we investigate here the partitioning of kinetic energy among the hyperspherical modes in an ensemble of reactive trajectories. The reactive trajectories are defined essentially in the same way as in the transition-path formalisms ${ }^{56-61}$ as follows.

First, we need to determine the appropriate vicinities for the two isomers of the $\mathrm{M}_{6}$ cluster in configuration space. For this purpose, we use the gyration radii of the cluster based on the results of Ref. 28. Figure 3(a) shows a typical time evolution of the three gyration radii, $a_{1}, a_{2}$, and $a_{3}$, of the $\mathrm{M}_{6}$ cluster at the total energy $E=-11.0 \varepsilon$. We see that the values 

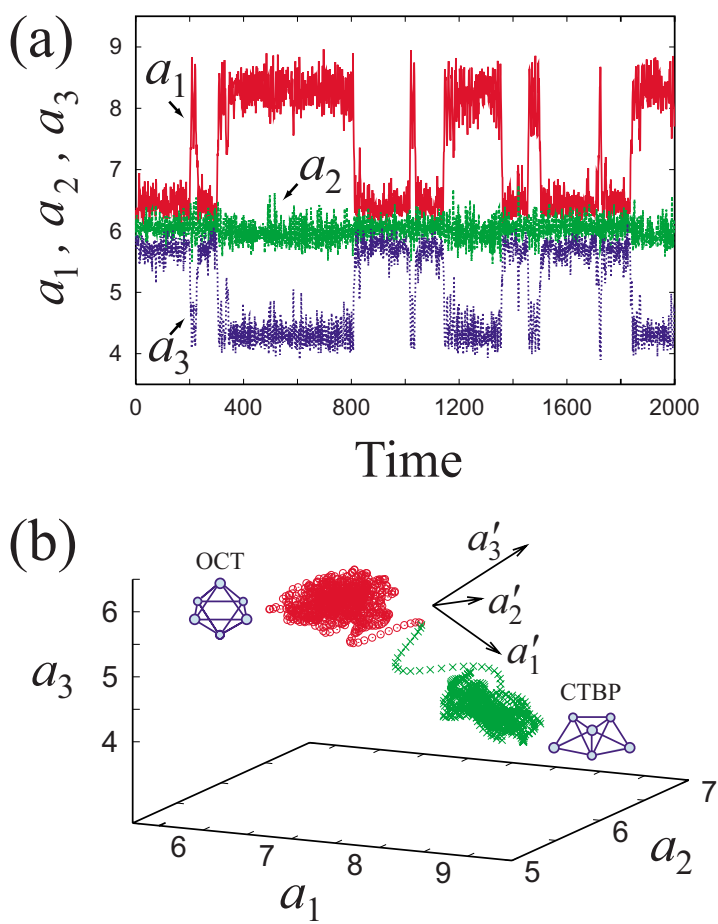

FIG. 3. (Color online) (a) A typical time evolution of the three gyration radii, $a_{1} \geq a_{2} \geq a_{3}$, at total energy $E=-11.0 \varepsilon$. In the time interval shown in the figure, the system had undergone structural transitions seven times from OCT to CTBP and six times from CTBP to OCT. (b) The projection of a trajectory with the total energy $E=-11.0 \varepsilon$ onto the three-dimensional space of gyration radii. The points plotted with circles are attributed to the potential well of OCT, while those plotted with crosses are attributed to the potential well of CTBP by the quenching method. The trajectory had undergone structural transition once. The three axes labeled as $a_{1}^{\prime}, a_{2}^{\prime}$, and $a_{3}^{\prime}$ in (b) represent the orthonormal frame $\mathbf{G}$, which will be introduced through the principal component analysis in Eq. (30). The axis labeled as $a_{1}^{\prime}$ represents the reactive mode of this study (see Sec. III B).

of gyration radii switch between the vicinities of the two sets of values at the equilibrium points, i.e., $\left(a_{1}, a_{2}, a_{3}\right)$ $=(5.97,5.97,5.97)$ for OCT and $\left(a_{1}, a_{2}, a_{3}\right)$ $=(8.19,5.89,4.27)$ for CTBP, reflecting the occurrence of structural transition between the two isomers. This result shows that the values of gyration radii can serve as useful order parameters to define the vicinities of the two isomers in the configuration space.

Figure 3(b) also shows the usefulness of gyration radii in defining the vicinities of the two isomers. In this figure, a trajectory with total energy $E=-11.0 \varepsilon$ is projected onto the three-dimensional space of gyration radii. The points plotted with circles are in the potential well of OCT, while those plotted with crosses are in the potential well of CTBP. Here we used the quenching method ${ }^{48}$ for this assignment of potential wells. We see that the three-dimensional space of gyration radii clearly distinguishes the two isomer regions and characterizes the occurrence of a single structural transition. Based on Figs. 3(a) and 3(b), we tentatively use $a_{1}$ as the order parameter to define the vicinities of the isomers. We then regard the region $a_{1} \leq 6.9$ as the isomer region of OCT and the region $a_{1} \geq 8.0$ as the isomer region for CTBP. The region in between these two isomer regions, where $6.9<a_{1}$ $<8.0$, is regarded as a transition region. Then, the reactive trajectories from OCT to CTBP are defined as the segments
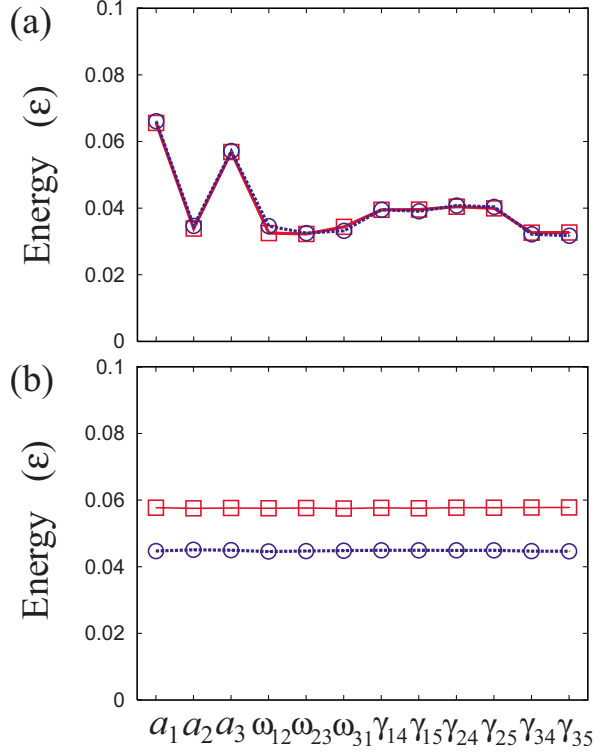

FIG. 4. (Color online) (a) Distribution of kinetic energy over the 12 hyperspherical modes of the $\mathrm{M}_{6}$ cluster computed by averaging over the reactive trajectories in the transition region at total energy $E=-11.0 \varepsilon$. The plot with squares corresponds to the reactive trajectories from OCT to CTBP, while the plot with circles corresponds to the reactive trajectories from CTBP to OCT. (b) Distribution of kinetic energy among the 12 hyperspherical modes in the isomer regions (potential wells) averaged over arbitrarily chosen long-time trajectories. The plot with squares represents the distribution of kinetic energy in the isomer region of OCT, while the plot with circles represents the distribution of kinetic energy in the isomer region of CTBP.

of trajectories in the transition region that depart from the isomer region of OCT and arrives at the isomer region of CTBP without returning to the initial isomer region of OCT. The reactive trajectories from CTBP to OCT are defined in the same way. A trajectory in the transition region that departs from one isomer region and returns to the same isomer region before entering into the other isomer region is regarded as a nonreactive trajectory.

Using the reactive trajectories defined as above, we now investigate the partitioning of kinetic energy among the hyperspherical modes. Figure 4(a) shows the distribution of kinetic energy among the 12 hyperspherical modes averaged over an ensemble of reactive trajectories in the transition region. Here, the kinetic energy in each hyperspherical mode is defined as the corresponding term of Eq. (9) as is prescribed in Sec. II. As is clearly seen from Fig. 4(a), the kinetic energy is not equipartitioned among the hyperspherical modes, but the $a_{1}$ - and $a_{3}$-modes have markedly more (about 1.5-2 times) kinetic energy than other internal modes. The kinetic energy partitioning for the reactions from OCT to CTBP is essentially the same as that from CTBP to OCT. This is due to the time-reversal symmetry of the dynamics. The result of Fig. 4(a) indicates that the two gyration-radius modes $a_{1}$ and $a_{3}$ need to be activated in order for the cluster to achieve structural transitions in both directions. Thus, the gyration-radius modes are the predominant modes according to our criterion for predominance as mentioned at the beginning of this subsection.

On the other hand, Fig. 4(b) shows the average partitioning of kinetic energy among the 12 hyperspherical modes over arbitrarily chosen long-time trajectories in the two iso- 
mer regions. Open squares represent the average partitioning of kinetic energy in the isomer region of OCT, while the open circles represent that in the isomer region of CTBP. It is clearly seen that kinetic energy is equipartitioned among the 12 hyperspherical modes in both the OCT and CTBP regions. By comparing the results of Figs. 4(a) and 4(b), we see that for the onset of structural transitions it is crucial to create the biased energy distribution as in Fig. 4(a) from the equipartitioned one as in Fig. 4(b) in each isomer region. The equipartition of kinetic energy in the isomer regions [Fig. 4(b)] suggests that the dynamics inside each isomer region is statistical as far as the ensemble of arbitrarily chosen longtime trajectories are concerned. However, this does not necessarily mean that the energy distribution among the internal modes evolves completely randomly in the isomer regions in the short time intervals right before the onset of structural transitions. Indeed, we show in Sec. IV that the distribution of kinetic energy over the internal modes evolves in quite characteristic manners in respective isomer regions before the onset of structural transitions.

The importance of the activation of the two gyrationradius modes, $a_{1}$-mode and $a_{3}$-mode, in the structural transitions of the $\mathrm{M}_{6}$ cluster may be understood by looking at Figs. 2(a), 2(c), 2(n), and 2(p): In order for the cluster to change its structure from OCT to CTBP, the cluster needs to acquire sufficient momentum or energy to elongate in the direction of the $a_{1}$-mode [Fig. 2(a)] and to contract in the direction of the $a_{3}$-mode [inverse of Fig. 2(c)]. On the other hand, in order for the cluster to change its structure from CTBP to OCT, the cluster needs to acquire sufficient momentum or energy to contract in the direction of the $a_{1}$-mode [inverse of Fig. 2(n)] and to elongate in the direction of the $a_{3}$-mode [Fig. 2(p)]. From other pictures in Fig. 2, one might see that activation of the twisting modes $\left(\omega_{i j}\right.$-modes $)$ and the shearing modes $\left(\gamma_{i k}\right.$-modes $)$ do not directly contribute to the structural transitions between the two isomers.

In our previous study, ${ }^{28}$ we observed numerically that the three gyration-radius modes change slowly in time compared to other hyperangular modes. This time-scale separation supported the idea of using the gyration radii as the predominant collective variables. ${ }^{67,68}$ The result of Fig. 4(a) of this study could be seen as another dynamical evidence for the predominance of gyration radii in structural transitions of the cluster. It is generally expected that the gyrationradius modes need to be activated in order for a molecular system to undergo reactions that accompanies a significant change in the mass distribution. This is because only the gyration-radius modes are directly linked to the dynamics of the overall mass distribution of a system with respect to the principal-axis frame, while all other hyperspherical modes, the twisting modes, and the shearing modes, are by definition associated only with the motions under the conditions of fixed overall mass distribution. (Note, however, that the twisting modes and the shearing modes can still influence and drive the dynamics of the overall mass distribution of a system indirectly via the mode coupling with the gyrationradius modes as we scrutinize in Sec. IV.)

\section{B. Reactive mode and reaction coordinate}

While the result of Fig. 4(a) has indicated that the two of the gyration-radius modes, $a_{1}$-mode and $a_{3}$-mode, are predominant in the structural transition of the $\mathrm{M}_{6}$ cluster, it is generally expected that the number of reactive modes can be reduced to 1 at least in the vicinity of the rank one saddle point. ${ }^{62-66}$ In addition, it is convenient to have only one reactive mode rather than two for the study of the mechanism for structural transition in Sec. IV. These considerations motivate us to transform here the three gyration-radius modes into one reactive mode and two bath modes based on the principal component analysis. ${ }^{69,70}$ The basic idea here is to find appropriate coordinate axes in the space of gyration radii, as shown in Fig. 3(b), so that one of the axes captures the structural transitions as clearly as possible.

First, we introduce a $3 \times 3$ variance-covariance matrix $\mathbf{C}$ whose components are defined by

$$
C_{i j}=\left\langle\left(a_{i}-\left\langle a_{i}\right\rangle\right)\left(a_{j}-\left\langle a_{j}\right\rangle\right)\right\rangle \quad(i, j=1,2,3),
$$

where the brackets $\langle\cdot\rangle$ represent the long-time average of a quantity. It is important to note that the three-dimensional space of gyration radii, as shown in Fig. 3(b), is Euclidean, although the $(3 n-6)$-dimensional internal space of an $n$-atom system as a whole is generally non-Euclidean. ${ }^{22-25}$ This Euclidean nature of the space of gyration radii can be seen from the expression for kinetic energy in Eq. (9), where the metric components for gyration radii are diagonal and unity. As a result, one can measure variance and covariance of the three gyration radii based simply on the standard Euclidean norm as in Eq. (28). The time period for this averaging should be long enough so that the system undergoes structural transitions back and forth for a sufficient number of times. Since the matrix $\mathbf{C}$ is symmetric, this matrix can be diagonalized by a $3 \times 3$ orthogonal matrix $\mathbf{G}$ through a similarity transformation, $\mathbf{G}^{T} \mathbf{C G}$. The first column of the matrix $\mathbf{G}$, which is defined as the eigenvector corresponding to the largest eigenvalue of $\mathbf{C}$, captures the direction in which the trajectory migrates the most widely in the space of gyration radii [see Fig. 3(b)]. The second and third columns of the matrix $\mathbf{G}$ are defined as the eigenvectors corresponding to the second largest and smallest eigenvalues of $\mathbf{C}$, respectively. By using the matrix $\mathbf{G}$, we can transform the original gyration radii $\boldsymbol{a} \equiv\left(a_{1}, a_{2}, a_{3}\right)^{T}$ into new ones $\boldsymbol{a}^{\prime}$ $\equiv\left(a_{1}^{\prime}, a_{2}^{\prime}, a_{3}^{\prime}\right)^{T}$ as

$$
\boldsymbol{a}^{\prime}=\mathbf{G}^{T} \boldsymbol{a} \text {. }
$$

The new gyration radius $a_{1}^{\prime}$ is expected to serve as the reaction coordinate that describes the structural transition better than $a_{1}$, while $a_{2}^{\prime}$ and $a_{3}^{\prime}$ are expected to be the less important coordinates, or simply the bath coordinates as we see below.

As an explicit expression for the matrix $\mathbf{G}$, we obtained

$$
\mathbf{G}=\left(\begin{array}{ccc}
0.801 & -0.292 & 0.523 \\
-0.045 & 0.841 & 0.539 \\
-0.597 & -0.455 & 0.660
\end{array}\right)
$$

by diagonalizing the matrix, Eq. (28), based on an ensemble of trajectories with total energy $E=-11.0 \varepsilon$. The directions of the first, second, and third column vectors of $\mathbf{G}$ in Eq. (30) 


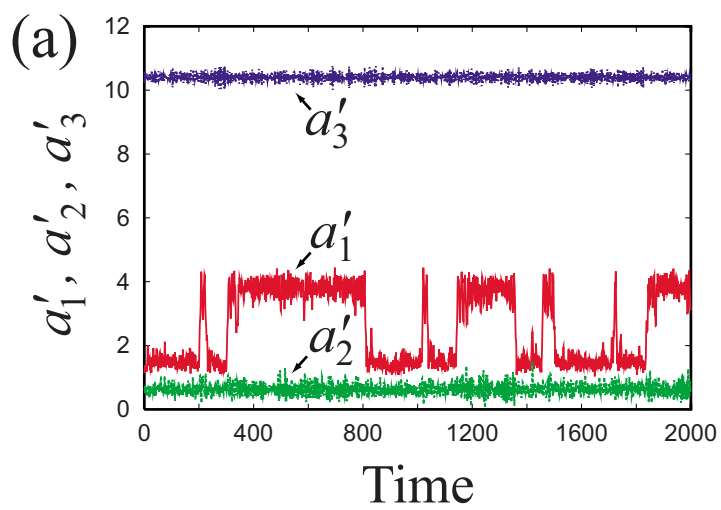

(b)

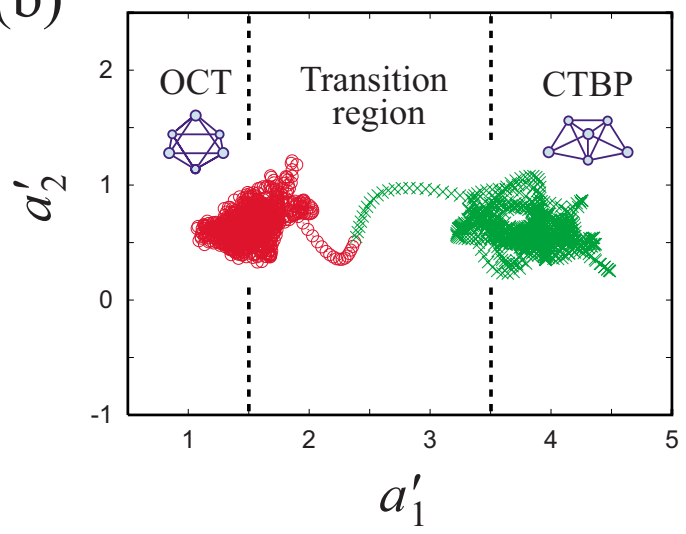

FIG. 5. (Color online) (a) Time evolution of the transformed gyration radii $a_{1}^{\prime}, a_{2}^{\prime}$, and $a_{3}^{\prime}$ for the same trajectory as in Fig. 3(a). (b) The projection of the trajectory in Fig. 3(b) onto the two-dimensional space of the transformed gyration radii $a_{1}^{\prime}$ and $a_{2}^{\prime}$. The points plotted with circles are attributed to the potential well of OCT, while those plotted with crosses are attributed to the potential well of CTBP by the quenching method. The coordinate $a_{1}^{\prime}$ serves as the reaction coordinate of the present study. The region in between the broken lines, where $1.5<a_{1}^{\prime}<3.5$, is defined as the transition region.

are depicted in Fig. 3(b) with labels $a_{1}^{\prime}, a_{2}^{\prime}$, and $a_{3}^{\prime}$, respectively. Equation (30) as well as Fig. 3(b) indicate that the first gyration radius $a_{1}$ contributes the most to $a_{1}^{\prime}$ as can be seen from the fact that the magnitude of the (1,1)-component of $\mathbf{G}$ is the largest among the components in the first column. Similarly, the third gyration radius $a_{3}$ contributes to $a_{1}^{\prime}$ the second most, while $a_{2}$ contributes the least to $a_{1}^{\prime}$. We also see that $a_{2}^{\prime}$ overlaps largely with $a_{2}$.

Figure 5(a) shows the time evolution of the three new coordinates, $a_{1}^{\prime}, a_{2}^{\prime}$, and $a_{3}^{\prime}$, introduced in Eq. (29), for the same trajectory as in Fig. 3(a). While the two variables, $a_{1}$ and $a_{3}$, change significantly upon structural transition in Fig. 3(a), only one variable $a_{1}^{\prime}$ changes significantly in Fig. 5(a). This indicates that the principal component analysis has successfully transformed the original three gyration radii into the single predominant coordinate $a_{1}^{\prime}$, which captures the structural transitions clearly, and other two coordinates, $a_{2}^{\prime}$ and $a_{3}^{\prime}$. Figure $5(\mathrm{~b})$ shows the projection of the same trajectory as in Fig. 3(b) onto the two-dimensional space of $a_{1}^{\prime}$ and $a_{2}^{\prime}$. We see that there are two regions where the trajectory is densely populated. In the left region, all the points on the trajectory (plotted with circles) are quenched to the bottom of the potential well of OCT. On the other hand, in the region on the right, all the points on the trajectory (plotted with crosses) are quenched to the bottom of the potential well of
CTBP. The two potential wells are clearly separated, and the system undergoes structural transition along the coordinate $a_{1}^{\prime}$ by passing through the region in between these two wells. Thus, $a_{1}^{\prime}$ can be regarded as the reaction coordinate. In Sec. IV, we use the reaction coordinate $a_{1}^{\prime}$ as well as the twodimensional space of $a_{1}^{\prime}$ and $a_{2}^{\prime}$ as in Fig. 5(b) frequently to describe the mechanisms for structural transitions of the cluster.

Since $a_{1}^{\prime}$ is a better reaction coordinate than $a_{1}$, we now refine the definitions of the reactive trajectories using $a_{1}^{\prime}$ based on Fig. 5(b): First, the transition region is defined as the region where $1.5<a_{1}^{\prime}<3.5$. Then the reactive trajectories from OCT to CTBP are defined as the segments of trajectories that start from the region $a_{1}^{\prime} \leq 1.5$ and arrives in the region $a_{1}^{\prime} \geq 3.5$ without returning to the initial region $a_{1}^{\prime}$ $\leq 1.5$. Similarly, the reactive trajectories from CTBP to OCT are defined as the segments of trajectories that start from the region $a_{1}^{\prime} \geq 3.5$ and arrives in the region $a_{1}^{\prime} \leq 1.5$ without returning to the initial region $a_{1}^{\prime} \geq 3.5$. Computations in Sec. IV will be performed using these definitions of reactive trajectories.

We next introduce the new modes and new kinetic energies that correspond to the new coordinates $a_{1}^{\prime}, a_{2}^{\prime}$, and $a_{3}^{\prime}$ introduced above. Since the matrix $\mathbf{G}$ is a constant matrix, the velocity of gyration radii $\dot{\boldsymbol{a}} \equiv\left(\dot{a}_{1}, \dot{a}_{2}, \dot{a}_{3}\right)^{T}$, is transformed into the new velocity, $\dot{\boldsymbol{a}}^{\prime}=\left(\dot{a}_{1}^{\prime}, \dot{a}_{2}^{\prime}, \dot{a}_{3}^{\prime}\right)^{T}$ in a similar way to Eq. (29) as

$$
\dot{\boldsymbol{a}}^{\prime}=\mathbf{G}^{T} \dot{\boldsymbol{a}} \text {. }
$$

Similarly, the corresponding basis vectors, $\mathbf{V}_{a_{1}}, \mathbf{V}_{a_{2}}$, and $\mathbf{V}_{a_{3}}$, defined in Eq. (20), which represent the gyration-radius modes, are transformed as

$$
\left(\begin{array}{c}
\mathbf{v}_{a_{1}^{\prime}} \\
\mathbf{v}_{a_{2}^{\prime}} \\
\mathbf{v}_{a_{3}^{\prime}}
\end{array}\right)=\mathbf{G}^{T}\left(\begin{array}{c}
\mathbf{v}_{a_{1}} \\
\mathbf{v}_{a_{2}} \\
\mathbf{v}_{a_{3}}
\end{array}\right),
$$

which is a symbolic expression of $\mathbf{V}_{a_{i}^{\prime}}=\sum_{j=1}^{3} G_{j i} \mathbf{V}_{a_{j}}$, where $G_{j i}$ is the $j i$-component of the matrix $\mathbf{G}$. These new basis vectors, $\mathbf{V}_{a_{1}^{\prime}}, \mathbf{V}_{a_{2}^{\prime}}$, and $\mathbf{V}_{a_{3}^{\prime}}$, represent the transformed gyrationradius modes, the $a_{1}^{\prime}, a_{2^{-}}^{\prime}$, and $a_{3^{\prime}}^{\prime}$-modes. Equations (31) and (32) ensure the relation,

$$
\sum_{i=1}^{3} \dot{a}_{i} \mathbf{V}_{a_{i}}=\sum_{i=1}^{3} \dot{a}_{i}^{\prime} \mathbf{V}_{a_{i}^{\prime}} .
$$

Finally, the expression for the kinetic energy of gyration radii in Eq. (9) is transformed as

$$
\frac{1}{2}\left(\dot{a}_{1}^{2}+\dot{a}_{2}^{2}+\dot{a}_{3}^{2}\right)=\frac{1}{2}\left(\dot{a}_{1}^{\prime 2}+\dot{a}_{2}^{\prime 2}+\dot{a}_{3}^{\prime 2}\right) .
$$

Each term on the right-hand side of this equation represents the kinetic energy in the respective $a_{i}^{\prime}$-mode $(i=1,2,3)$.

Figure 6 shows the new gyration-radius modes, $a_{1^{-}}^{\prime}, a_{2}^{\prime}$, and $a_{3}^{\prime}$-modes, defined by Eq. (32) with the matrix $\mathbf{G}$ in Eq. (30) for the two isomers of the $M_{6}$ cluster. The representation schemes in Fig. 6 are the same as in Fig. 2. Note that the coordinates, $a_{1}^{\prime}, a_{2}^{\prime}$, and $a_{3}^{\prime}$, in Fig. 5 are essentially the variables that quantify the degree of deformation of the cluster in the directions represented by the respective modes in Fig. 6 . 
(a)

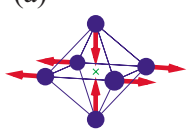

$a_{1}^{\prime}$-mode

(d)

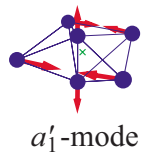

(b)

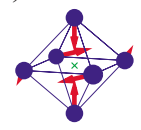

$a_{2}^{\prime}-$ mode

(e)

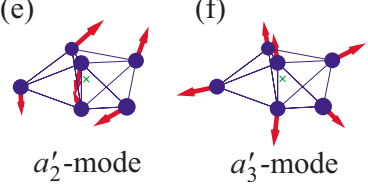

(c)

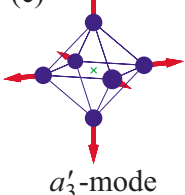

(f)
FIG. 6. (Color online) The transformed gyration-radius modes, $a_{1^{-}}^{\prime}, a_{2^{-}}^{\prime}$, and $a_{3}^{\prime}$-modes, defined by Eq. (32) with Eq. (30) for the OCT isomer [(a)-(c)] and for the CTBP isomer [(d)-(f)]. In (d), the $a_{1}^{\prime}$-mode is shown in the negative direction (the vector $-\mathbf{V}_{a_{1}^{\prime}}$ ) so that the relevance of the $a_{1}^{\prime}$-mode to the structural transition from CTBP to OCT becomes transparent. The representation schemes are the same as in Fig. 2. The $a_{1}^{\prime}$-mode [shown in (a) and (d)] serves as the reactive mode for both the reaction from OCT to CTBP and the reaction from CTBP to OCT.

We see from Fig. 6(a) that the activation of the $a_{1}^{\prime}$-mode around the OCT isomer facilitates the vibration of the cluster to elongate/contract in the direction of the first principal axis and contract/elongate in the direction of the third principal axis in the same phase, which can in turn induce the structural transition into the CTBP isomer. Similarly, the activation of the $a_{1}^{\prime}$-mode around the CTBP isomer shown in Fig. 6(d) facilitates the vibrations to contract/elongate in the direction of the first principal axis and elongate/contract in the direction of the third principal axis in the same phase, which can induce the structural transition into the OCT isomer. Note that, in Fig. 6(d), we have plotted the $a_{1}^{\prime}$-mode in the negative direction (the vector $-\mathbf{V}_{a_{1}^{\prime}}$ ) so that the relevance of the $a_{1}^{\prime}$-mode to the structural transition from CTBP to OCT becomes transparent. Thus, the $a_{1}^{\prime}$-mode is expected to serve as the reactive mode for both the reaction from OCT to CTBP and for the reaction from CTBP to OCT. On the other hand, the $a_{3}^{\prime}$-mode shown in Figs. 6(c) and 6(f), which is regarded as the least important gyration-radius mode according to the principal component analysis, possesses the tendency of isotropic inflation and contraction of the system. Since the isotropic inflation and contraction are not relevant for the structural transition between the OCT and CTBP, we see that the $a_{3}^{\prime}$-mode serves as a bath mode.

Figure 7 (a) shows the distribution of kinetic energy among the 12 internal modes averaged over the reactive trajectories in the transition region. Here, the first three modes are the transformed gyration-radius modes, $a_{1^{-}}^{\prime}, a_{2^{-}}^{\prime}$, and $a_{3}^{\prime}$-modes, whose kinetic energies are defined as the respective terms on the right-hand side of Eq. (34). Kinetic energies in all other modes, the twisting modes $\omega_{i j}$ and the shearing modes $\gamma_{i k}$, are defined in the same way as in Fig. 4 based on Eq. (9). Open squares represent the distribution of kinetic energy averaged over the reactive trajectories from OCT to CTBP, while open circles represent the distribution of kinetic energy averaged over the reactive trajectories from CTBP to OCT. While the two gyration-radius modes, $a_{1}$ and $a_{3}$, have a large amount of kinetic energy in the transition region in Fig. 4(a), here in Fig. 7(a), only the $a_{1}^{\prime}$-mode has a large
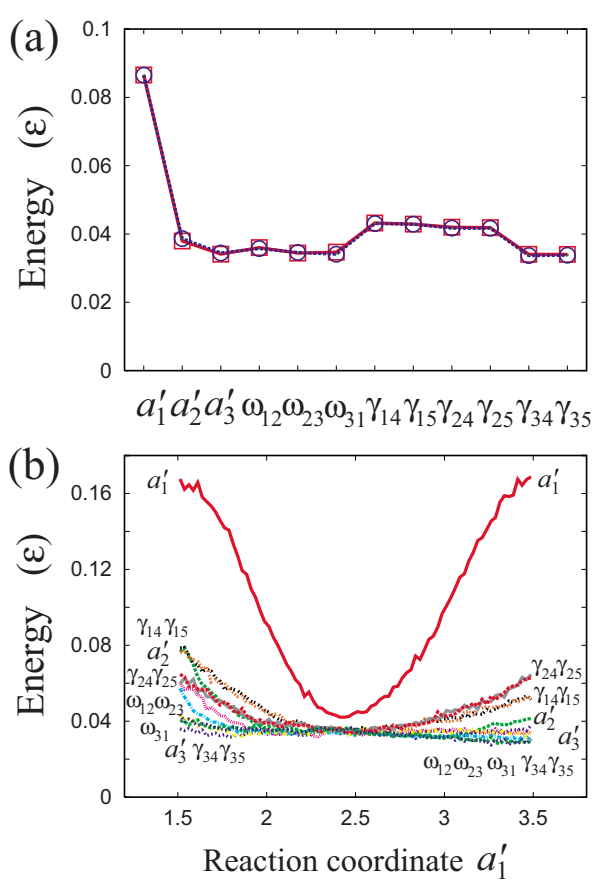

FIG. 7. (Color online) (a) The distribution of kinetic energy among the 12 hyperspherical modes of the six-atom cluster computed by averaging over the reactive trajectories in the transition region at total energy $E=-11.0 \varepsilon$. The three gyration-radius modes are the transformed ones, $a_{1^{-}}^{\prime}, a_{2^{-}}^{\prime}$, and $a_{3}^{\prime}$-modes. The plot with squares corresponds to the reaction from OCT to CTBP, while the plot with circles corresponds to the reaction from CTBP to OCT. (b) shows the change in the kinetic energy in each mode along the reaction coordinate $a_{1}^{\prime}$ computed by averaging over reactive trajectories in the transition region. The top curve represents the kinetic energy in the reactive mode $a_{1}^{\prime}$. All other curves represent the kinetic energies in the remaining hyperspherical modes as indicated.

amount of kinetic energy. This indicates that the predominant $a_{1}$ - and $a_{3}$-modes are successfully merged into the single reactive mode $a_{1}^{\prime}$, and all other 11 modes can be regarded as bath modes at least in the transition region.

Figure 7(b) shows the partitioning of kinetic energy along the reaction coordinate $a_{1}^{\prime}$ computed by averaging over an ensemble of the reactive trajectories from CTBP to OCT. Due to the time-reversal symmetry, the partitioning of kinetic energy averaged over an ensemble of reactive trajectories from OCT to CTBP is essentially the same as the one in Fig. 7(b) (data not shown). The left and right ends of the curves (at $a_{1}^{\prime}=1.5$ and $a_{1}^{\prime}=3.5$ ) correspond to the borders of the transition region at the OCT side and the CTBP side, respectively. We see that the $a_{1}^{\prime}$-mode has markedly a larger amount of kinetic energy than other 11 internal modes over the whole range of the reaction coordinate. The kinetic energy in the $a_{1}^{\prime}$-mode is the largest at both ends of the transition region and reaches a minimum value at around $a_{1}^{\prime}=2.4$, which roughly corresponds to the top of the potential energy barrier in the saddle region. The kinetic energies in all other bath modes also decrease toward to center of the figure, and they tend to be constant in the saddle region along the reaction coordinate. Thus, it is fair to speculate that a large amount of kinetic energy in the reactive mode at both ends of the figure is consumed to surmount the potential energy barrier without dissipating into other bath modes largely. This result indicates that the reaction coordinate and the bath modes are 
fairly decoupled. It could be another evidence for the regularity of the structural transition dynamics in the saddle region, which has been predicted and assessed from the viewpoint of phase space structures. ${ }^{52,62-66}$

Thus, in this section, we have constructed the reaction coordinate $a_{1}^{\prime}$ and the corresponding reactive mode, $a_{1}^{\prime}$-mode. It should be noted that the reaction coordinate and the reactive mode are complementary concepts that characterize the same molecular degree of freedom. In general, a mode defines the directionality of deformation (vibration) of a system while a coordinate quantifies the amount of deformation in each mode. In Sec. IV, we investigate the partitioning of kinetic energy in terms of the modes (Sec. IV A), whereas we investigate equations of motion (Sec. IV B) and the driving mechanisms for structural transitions (Sec. IV C) in terms of the coordinates. All other 11 hyperspherical modes, the $a_{2}^{\prime}$-mode, $a_{3}^{\prime}$-mode, the three twisting modes $\left\{\omega_{i j}\right\}$, and the six shearing modes $\left\{\gamma_{i k}\right\}$, can be regarded as bath modes at least in the transition region.

\section{INTRAMOLECULAR ENERGY TRANSFER AND THE DRIVING MECHANISMS FOR STRUCTURAL TRANSITIONS}

While Sec. III has focused on the dynamics of the cluster in the transition region to construct the reactive mode and the reaction coordinate $a_{1}^{\prime}$, we study here the dynamics of the cluster inside the two potential wells to clarify the driving mechanisms for structural transitions. We highlight the mechanisms for the energy transfer from bath modes into the reactive mode $a_{1}^{\prime}$ by investigating the coupling between the reactive mode and the bath modes in the respective potential wells.

\section{A. Energy transfer from the bath modes into the reactive mode}

We first investigate the dynamics in the potential well of the OCT isomer to clarify how a large amount of kinetic energy is transferred from the bath modes into the reactive mode $a_{1}^{\prime}$ right before the system starts a structural transition into CTBP. In order to clarify this energy transfer process, we investigate the "past" time evolution of kinetic-energy distribution among the 12 hyperspherical modes in the OCT isomer in the time course of reactive trajectories right before they start structural transition. Since each time evolution of kinetic-energy distribution possesses large fluctuations, we average the time evolutions over an ensemble of reactive trajectories so that the general tendencies of energy transfer become clear. This can be done as follows.

One first collects an ensemble of reactive trajectories that undergo structural transition from OCT to CTBP (see Sec. III B for the definition of reactive trajectories). For each trajectory, the time origin, $t=0$, is reset to the last moment that the trajectory crosses the border of the transition region, the line $a_{1}^{\prime}=1.5$ in Fig. 5(b), from the left to the right to start the structural transition into CTBP. We then computed average kinetic-energy partitioning over these trajectories at different time instances in the negative time domain $(t \leq 0)$ before the system starts structural transition.

Figures 8(a) and 8(b) show the time evolution of the computed average kinetic-energy distribution in the OCT isomer prior to the onset of structural transition. The schematic diagram above Fig. 8(a) shows the time domains of the plots in the panels (a) and (b). The plot with diamonds in Fig. 8(b) corresponds to the average kinetic-energy distribution at time $t=-0.9$ prior to the onset of structural transition. As time goes on, the average energy distribution evolves in the order of the plots with inverted triangles $(t=-0.8)$, triangles $(t=-0.7)$, circles $(t=-0.6)$, and squares $(t=-0.5)$. Figure 8(a) is the continuation of Fig. 8(b), where the average kineticenergy distribution changes in the order of the plots with diamonds $(t=-0.4)$, inverted triangles $(t=-0.3)$, triangles $(t$ $=-0.2)$, and circles $(t=-0.1)$. The average energy distribution shown with squares in Fig. 8(a) is the final distribution at time $t=0$. As is expected, the reactive mode $a_{1}^{\prime}$ has a large amount of kinetic energy at $t=0$, after which the reactive trajectories undergo structural transition from OCT to CTBP by consuming the kinetic energy in the reactive mode.

From the results in Figs. 8(a) and 8(b), we see that the three twisting modes, $\omega_{12}, \omega_{23}$, and $\omega_{31}$, as well as the $a_{2}^{\prime}$-mode, exchange kinetic energy actively with the reactive mode $a_{1}^{\prime}$ before the reactive mode acquires sufficient energy to get out of the OCT region. In particular, the three twisting modes and the $a_{2}^{\prime}$-mode are highly active at the time around $t=-0.4$ to -0.5 . Thus, the activation of these four modes is expected to serve as a precursor of the structural transition from the OCT isomer.

We next investigate the energy transfer processes in the CTBP isomer prior to the onset of structural transition into OCT. Similarly to the procedure to obtain Figs. 8(a) and 8(b), we collected an ensemble of reactive trajectories that undergo structural transition from CTBP to OCT. For each trajectory, the time origin, $t=0$, is reset to the last moment that the trajectory crosses the border of the transition region, the line $a_{1}^{\prime}=3.5$ in Fig. 5(b), from the right to the left to start structural transition into OCT. The kinetic energy distribution is then averaged over the ensemble of these trajectories at different time instances from $t=-3.6$ to $t=0$ before the system starts structural transition.

Figures 8(c) and 8(d) show thus computed time evolution of average kinetic energy distribution in CTBP. The schematic diagram above Fig. 8(c) shows the time domains of the plots in the panels (c) and (d). The time series of kinetic energy distribution starts from the one plotted with diamonds in Fig. 8(d) at time $t=-3.6$ and evolves in the order of the plots with inverted triangles $(t=-3.2)$, triangles $(t=-2.8)$, circles $(t=-2.4)$, and squares $(t=-2.0)$. Figure 8(c) continues from Fig. 8(d) in the order of the plots with diamonds $(t=-1.6)$, inverted triangles $(t=-1.2)$, triangles $(t=$ $-0.8)$, circles $(t=-0.4)$, and finally squares $(t=0)$. Similarly to Fig. 8(a), the reactive mode $a_{1}^{\prime}$ has a large amount of kinetic energy at $t=0$, after which the reactive trajectories undergo structural transition into OCT by consuming the kinetic energy in the reactive mode.

The time evolution of average kinetic energy distribution 

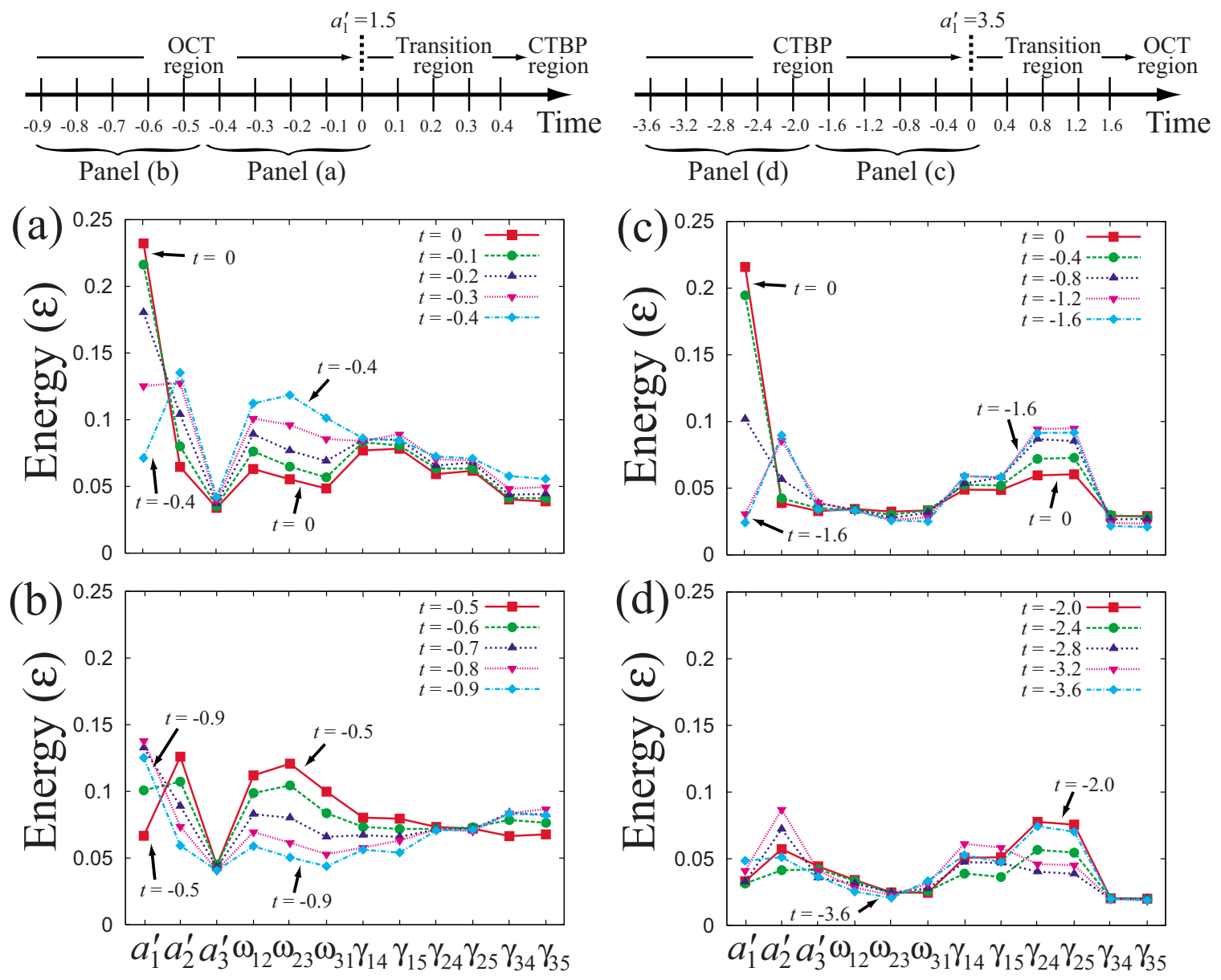

FIG. 8. (Color online) Panels (a) and (b) show the time evolution of average kinetic energy distribution among the 12 hyperspherical modes in the potential well of OCT prior to the onset of structural transition into CTBP at $t=0$. The distribution evolves in time in the order of the plot with diamonds $(t=-0.9)$, inverted triangles $(t=-0.8)$, triangles $(t=-0.7)$, circles $(t=-0.6)$, and squares $(t=-0.5)$ in $(\mathrm{b})$, and continues to the plot with diamonds $(t=-0.4)$, inverted triangles $(t=-0.3)$, triangles $(t=-0.2)$, circles $(t=-0.1)$, and squares $(t=0)$ in (a). The schematic above (a) shows the time domains of the plots in the panels (a) and (b). Panels (c) and (d) show the average time evolution of kinetic energy distribution over the 12 internal modes in the potential well of CTBP prior to the onset of structural transition into OCT at $t=0$. Time evolutions of the energy distribution in (c) and (d) are in the same order as in (a) and (b) but for the different time duration from $t=-3.6$ to $t=0$ as indicated. The schematic above (c) shows the time domains of the plots in panels (c) and (d).

in Figs. 8(c) and 8(d) in the CTBP isomer is quite different from the one in Figs. 8(a) and 8(b) in the OCT isomer: While the three twisting modes, $\omega_{12}, \omega_{23}$, and $\omega_{31}$, are highly active right before the activation of the reactive mode $a_{1}^{\prime}$ in OCT [see Figs. 8(a) and 8(b)], these three twisting modes are highly inactive in CTBP, having very low kinetic energy right before the activation of the reactive mode $a_{1}^{\prime}$ [see Figs. $8(\mathrm{c})$ and 8(d)]. Instead, the two shearing modes, $\gamma_{24}$ and $\gamma_{25}$, are active right before the reactive mode $a_{1}^{\prime}$ acquires a large amount of kinetic energy in CTBP. We scrutinize the mechanisms for such characteristic energy transfer processes prior to the onset of structural transitions in respective isomers in the following two subsections.

It should be noted that the time evolution of the average kinetic energy distribution right after the system has moved into each of the two isomers is essentially the reverse of the time evolution shown in Fig. 8. This is due to the timereversal symmetry of the dynamics. We have confirmed that the three twisting modes and the $a_{2}^{\prime}$-mode become active right after the system has moved into the OCT isomer (data not shown). On the other hand, in the CTBP isomer, the three twisting modes become inactive and the two shearing modes, $\gamma_{24}$ and $\gamma_{25}$, and the $a_{2}^{\prime}$-mode becomes active right after the system has moved into the CTBP isomer region (data not shown).

Another important fact that can be observed from Fig. 8 is that the timescale for intramolecular energy transfer is different in the two isomers. We see from Fig. 8 that the average distribution of kinetic energy evolves faster in the OCT isomer than in the CTBP isomer. For example, in the OCT isomer, it only takes about 0.4 time units (measured in absolute unit) for the kinetic energy in the reactive mode $a_{1}^{\prime}$ to increase from the minimum to the maximum in Fig. 8(a). In the CTBP isomer, on the other hand, it takes about 1.6 time units (measured in absolute unit) for the similar increment of kinetic energy in the reactive mode $a_{1}^{\prime}$. Thus, it is fair to speculate that intramolecular energy transfer is faster in the OCT isomer than in the CTBP isomer. Such timescales for energy transfer can be closely related to the timescales for mixing in the phase space, which can in turn influence the statistical nature of the reaction rate processes. We wish to scrutinize the issues on the timescales of energy transfer and reaction rates in a separate publication. 


\section{B. Characterization of mode coupling}

For the purpose of understanding the mechanisms behind the characteristic energy transfer processes right before the onset of structural transitions observed in Fig. 8, we characterize here the forces that are responsible for the coupling between the reactive mode, $a_{1}^{\prime}$-mode, and other bath modes. To this end, in this subsection, we mainly work on coordinates instead of modes.

Our reaction coordinate $a_{1}^{\prime}$ is a linear combination of the three gyration radii as defined in Eq. (29), and the equations of motion for gyration radii are given in Ref. 28 as

$$
\begin{aligned}
& \ddot{a}_{1}=\Gamma_{12} \omega_{12}^{2}+\Gamma_{13} \omega_{31}^{2}+a_{1}\left(\gamma_{14}^{2}+\gamma_{15}^{2}\right)-\frac{\partial V}{\partial a_{1}}, \\
& \ddot{a}_{2}=\Gamma_{21} \omega_{12}^{2}+\Gamma_{23} \omega_{23}^{2}+a_{2}\left(\gamma_{24}^{2}+\gamma_{25}^{2}\right)-\frac{\partial V}{\partial a_{2}}, \\
& \ddot{a}_{3}=\Gamma_{31} \omega_{31}^{2}+\Gamma_{32} \omega_{23}^{2}+a_{3}\left(\gamma_{34}^{2}+\gamma_{35}^{2}\right)-\frac{\partial V}{\partial a_{3}}
\end{aligned}
$$

for a six-atom system, where

$$
\Gamma_{i j}=\frac{a_{i}\left(a_{i}^{2}+3 a_{j}^{2}\right)\left(a_{i}^{2}-a_{j}^{2}\right)}{\left(a_{i}^{2}+a_{j}^{2}\right)^{2}} \quad(i, j=1,2,3, i \neq j) .
$$

Physical significance of each term in Eqs. (35)-(37) has been studied in detail in Ref. 28. In short, while the final (fourth) terms on the right-hand sides of these equations are the components of the ordinary potential force, all the other terms represent the internal centrifugal force. The latter force is termed centrifugal because each term of this force is proportional to the square of the quasivelocity, $\omega_{i j}$ or $\gamma_{i k}$, which is essentially the internal angular velocity. The six terms proportional to $\omega_{i j}^{2}$ in Eqs. (35)-(37) have a symmetry breaking effect on the mass distribution of a system. This is due to the relationship

$$
\Gamma_{12}, \Gamma_{13}, \Gamma_{23} \geq 0, \quad \Gamma_{21}, \Gamma_{31}, \Gamma_{32} \leq 0,
$$

so that the terms proportional to $\omega_{i j}^{2}$ always work positively on the largest gyration radius $a_{1}$ and negatively on the smallest gyration radius $a_{3}$. As a result, a system is always forced to elongate in the longest (most massive) direction and contract in the shortest (least massive) direction along principal axes. This explains the symmetry breaking effect of the terms proportional to $\omega_{i j}^{2}$ on the overall mass distribution of the system. The terms proportional to $\gamma_{i k}^{2}$ in Eqs. (35)-(37) have an inflating effect because these terms always act positively on all of the three gyration radii to inflate the overall mass distribution of the system. The internal centrifugal force is different from ordinary centrifugal forces because ordinary centrifugal forces come into play only when the system has finite angular momentum, while the internal centrifugal force arises even in the systems with zero total angular momentum. In fact, the internal centrifugal force originates from molecular vibrations themselves, more specifically, the twisting modes $\left\{\omega_{i j}\right\}$ and the shearing modes $\left\{\gamma_{i k}\right\}$, as can be seen from Eqs. (35)-(37). Thus the internal centrifugal force can mediate the mode coupling as well as the energy transfer among the gyration-radius modes and other internal modes (the twisting and shearing modes).

It is convenient to introduce a three-dimensional vector representation of Eqs. (35)-(37),

$$
\ddot{a}=f_{\mathrm{ICF}}+f_{\mathrm{Pot}},
$$

where $f_{\mathrm{ICF}}$ represents the terms of the internal centrifugal force (terms including $\left\{\omega_{i j}\right\}$ and $\left\{\gamma_{i k}\right\}$ ) and $\boldsymbol{f}_{\mathrm{Pot}}$ represents the terms of the potential force [the last terms of Eqs. (35)-(37)]. Since the gyration radii $\boldsymbol{a}=\left(a_{1}, a_{2}, a_{3}\right)^{T}$ are transformed into the new coordinates $\boldsymbol{a}^{\prime}=\left(a_{1}^{\prime}, a_{2}^{\prime}, a_{3}^{\prime}\right)^{T}$ by the multiplication of the constant matrix as $\boldsymbol{a}^{\prime}=\mathbf{G}^{T} \boldsymbol{a}$ [see Eq. (29)], where $\mathbf{G}$ is the matrix defined by Eq. (30), the equations of motion for the new coordinates can be written (since $\mathbf{G}$ is time independent) as

$$
\ddot{\boldsymbol{a}}^{\prime}=\mathbf{G}^{T} \boldsymbol{f}_{\mathrm{ICF}}+\mathbf{G}^{T} \boldsymbol{f}_{\mathrm{Pot}} .
$$

The terms $\mathbf{G}^{T} \boldsymbol{f}_{\text {ICF }}$ and $\mathbf{G}^{T} \boldsymbol{f}_{\text {Pot }}$ are the internal centrifugal force and the potential force represented in the new coordinates.

In order to characterize the effects of the internal centrifugal force and the potential force, we plotted the averaged field of $\mathbf{G}^{T} \boldsymbol{f}_{\text {ICF }}$ in Fig. 9(a) and that of $\mathbf{G}^{T} \boldsymbol{f}_{\text {Pot }}$ in Fig. 9(b) in the two-dimensional space of $a_{1}^{\prime}$ and $a_{2}^{\prime}$ at total energy $E=$ $-11.0 \varepsilon$. This two-dimensional space is the same as the one in Fig. 5(b), where the potential well of OCT is located in the left region of this space, and that of CTBP is located in the right region. Here the average is taken over an ensemble of arbitrarily chosen long-time trajectories with total energy $E$ $=-11.0 \varepsilon$ at each point of the space of $a_{1}^{\prime}$ and $a_{2}^{\prime}$. Figure 9(c) shows the superposition of the averaged force fields in Figs. 9(a) and 9(b). In Figs. 9(a)-9(c), the direction of each arrow represents the direction of the respective force at each point in the $a_{1}^{\prime}-a_{2}^{\prime}$ space, and the length of each arrow is proportional to the magnitude of the respective force at each point.

As can be seen from Fig. 9(a), the internal centrifugal force has a clear directionality from the OCT isomer region to the CTBP isomer region. This can be naturally understood from the fact that the internal centrifugal force has the general tendency to deform a spherical mass distribution of a system like OCT to the elongated ones like CTBP as noted above. In contrast to the internal centrifugal force, the potential force field has the directionality to the bottom of the potential well in each region of the two isomers as can be seen from Fig. 9(b): In this $a_{1}^{\prime}-a_{2}^{\prime}$ space, the point corresponding to the potential minimum of OCT is located at $\left(a_{1}^{\prime}, a_{2}^{\prime}\right)=(0.95,0.56)$, and that of CTBP is located at $\left(a_{1}^{\prime}, a_{2}^{\prime}\right)=(3.75,0.62)$. Thus, we see that the potential force has a general tendency to preserve the structure of each isomer in the respective potential well as is naturally expected.

It is important to note that the internal centrifugal force is much stronger in the OCT region than in the CTBP region [see Fig. 9(a)]. In general, the internal centrifugal force in molecular vibrations tends to be strong around the structures 
where the values of two or three of the gyration radii are close to each other. This property of the internal centrifugal force can be concisely explained as follows. First, we express the internal centrifugal force in terms of the kinetic energies in the twisting modes $K_{\omega_{i j}}$ and the shearing modes $K_{\gamma_{i j}}$, which are defined by the respective terms of Eq. (9). Using $K_{\omega_{i j}}$ and $K_{\gamma_{i j}}$, the squares of the quasivelocities of $\omega_{i j}^{2}$ and $\gamma_{i k}^{2}$ can be expressed as

$$
\omega_{i j}^{2}=\frac{2 K_{\omega_{i j}}\left(a_{i}^{2}+a_{j}^{2}\right)}{\left(a_{i}^{2}-a_{j}^{2}\right)^{2}}, \quad \gamma_{i k}^{2}=\frac{2 K_{\gamma_{i k}}}{a_{i}^{2}} .
$$

By applying Eq. (42) to the respective terms on the righthand sides of Eqs. (35)-(37), we obtain

$$
f_{\mathrm{ICF}}=\left(\begin{array}{l}
\frac{2 a_{1}\left(a_{1}^{2}+3 a_{2}^{2}\right)}{a_{1}^{4}-a_{2}^{4}} K_{\omega_{12}}+\frac{2 a_{1}\left(a_{1}^{2}+3 a_{3}^{2}\right)}{a_{1}^{4}-a_{3}^{4}} K_{\omega_{31}}+\frac{2}{a_{1}}\left(K_{\gamma_{14}}+K_{\gamma_{15}}\right) \\
\frac{2 a_{2}\left(a_{2}^{2}+3 a_{1}^{2}\right)}{a_{2}^{4}-a_{1}^{4}} K_{\omega_{12}}+\frac{2 a_{2}\left(a_{2}^{2}+3 a_{3}^{2}\right)}{a_{2}^{4}-a_{3}^{4}} K_{\omega_{23}}+\frac{2}{a_{2}}\left(K_{\gamma_{24}}+K_{\gamma_{25}}\right) \\
\frac{2 a_{3}\left(a_{3}^{2}+3 a_{1}^{2}\right)}{a_{3}^{4}-a_{1}^{4}} K_{\omega_{31}}+\frac{2 a_{3}\left(a_{3}^{2}+3 a_{2}^{2}\right)}{a_{3}^{4}-a_{2}^{4}} K_{\omega_{23}}+\frac{2}{a_{3}}\left(K_{\gamma_{34}}+K_{\gamma_{35}}\right)
\end{array}\right) .
$$

Here, the six terms containing the factors $K_{\omega_{i j}}$ have the symmetry breaking effect on the overall mass distribution of the system, while the other six terms containing the factors $K_{\gamma_{i k}}$ have the inflating effect on the overall mass distribution of the system as we discussed right after Eqs. (35)-(37). Since the kinetic energies $K_{\omega_{i j}}$ and $K_{\gamma_{i k}}$ are generally not zero but tend to be equal in the dynamics in potential wells after averaging [see Fig. 4(b)], magnitudes of the terms containing the factors $K_{\omega_{i j}}$ in Eq. (43) tend to be very large when $a_{i}$ and $a_{j}$ are close to each other due to the small denominators of the form $\left(a_{i}^{4}-a_{j}^{4}\right)$. Therefore, the internal centrifugal force (more specifically, the symmetry breaking effect of the internal centrifugal force) tends to be strong in the molecular vibrations near the structures where two or three of the gyration radii are close to each other. Therefore, since the three gyration radii are very close to each other in the OCT isomer of our $\mathrm{M}_{6}$ cluster [see Fig. 3(a)], the symmetry breaking effect of the internal centrifugal force is inevitably very strong in this isomer. This is the reason why the internal centrifugal force field in Fig. 9(a) is strong in the OCT isomer. On the other hand, since the values of the three gyration radii are apart in the CTBP isomer [see Fig. 3(a)], the internal centrifugal force is not very strong in this isomer.

In order to characterize the joint effects of the internal centrifugal force and the potential force, it is useful to introduce an integral curve of the sum of the averaged force fields along the reaction coordinate $a_{1}^{\prime}$, which is shown in Fig. 9(d). In obtaining the curve in Fig. 9(d), we first computed average force fields for both the internal centrifugal force and the potential force along $a_{1}^{\prime}$ using an ensemble of arbitrarily chosen long-time trajectories. These average force fields are summed and integrated along $a_{1}^{\prime}$. This integral curve, called mean force potential, is useful to quantify the net dynamical stability of each isomer. The mean force potential in Fig. 9(d) possesses two wells; the left well corresponds to the OCT isomer and the right one corresponds to the CTBP isomer.
Since the depths of the two wells are roughly equal, we see that the stabilities of OCT and CTBP are comparative. Indeed, the cluster stays in the two isomers for roughly the same amount of time at the total energy $E=-11.0 \varepsilon$ as we noted in Sec. II C. In Sec. IV C, we utilize this mean force potential to explain the driving mechanism for structural transition.

\section{Driving mechanisms for structural transitions: Roles of the twisting modes}

Based on the properties of the forces acting on the reaction coordinate presented in Sec. IV B, we now clarify the dynamical mechanisms behind the characteristic time evolutions of kinetic energy distribution among the internal modes right before the onset of structural transitions observed in Fig. 8. We primarily focus here on the questions of why the three twisting modes, $\omega_{12}, \omega_{23}$, and $\omega_{31}$, tend to be highly active right before the onset of structural transition in the OCT isomer [see Figs. 8(a) and 8(b)], and why the three twisting modes tend to be highly inactive right before the onset of structural transition in the CTBP isomer [see Figs. $8(\mathrm{c})$ and $8(\mathrm{~d})$ ]. This is because the activity of the twisting modes, in general, plays a major role for the onset of structural transitions between a symmetric and asymmetric structures such as the OCT and CTBP isomers of our $\mathrm{M}_{6}$ cluster as we see below.

Equation (43) exemplifies the fact that activity of the $\omega_{i j}$-modes (twisting modes) and $\gamma_{i k}$-modes (shearing modes) directly controls the strength of the internal centrifugal force acting on the gyration radii: For example, when the three twisting modes are active having a large amount of kinetic energy, i.e., $\left\{K_{\omega_{i j}}\right\}$ are large in Eq. (43), the symmetry breaking effect of the internal centrifugal force becomes strong. Therefore, the fact that the three twisting modes become highly active in the OCT isomer right before the onset of 

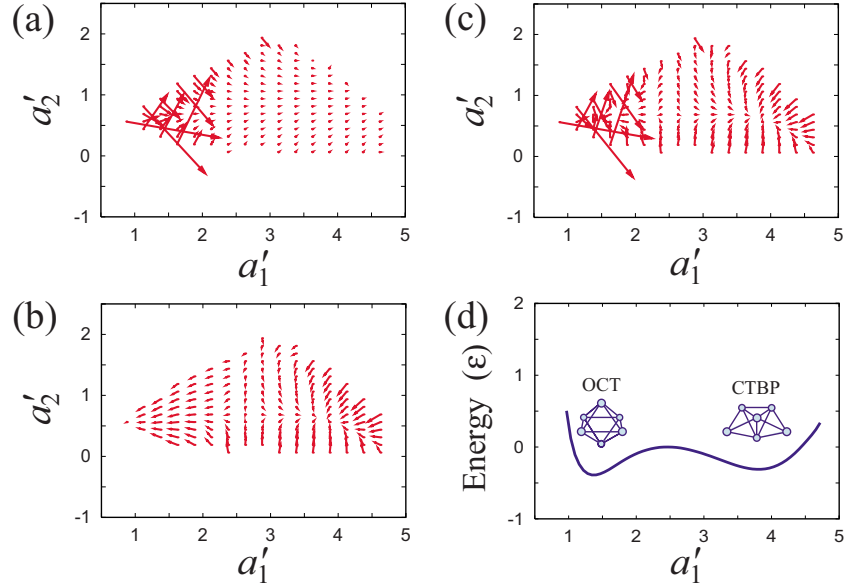

FIG. 9. (Color online) (a) Averaged field of the internal centrifugal force in the two-dimensional space of the reaction coordinate $a_{1}^{\prime}$ and $a_{2}^{\prime}$. (b) Averaged field of the potential force in the same space as in (a). In this space, the point corresponding to the potential minimum of OCT is located at $\left(a_{1}^{\prime}, a_{2}^{\prime}\right)=(0.95,0.56)$, and that of CTBP is located at $\left(a_{1}^{\prime}, a_{2}^{\prime}\right)=(3.75,0.62)$ In obtaining (a) and (b), average is taken over an ensemble of arbitrarily chosen long-time trajectories with total energy $E=-11.0 \varepsilon$. (c) is the superposition of (a) and (b). (d) shows the line integral (mean force potential) of the sum of the average internal centrifugal force and average potential force along the reaction coordinate $a_{1}^{\prime}$.

structural transition [see Figs. 8(a) and 8(b)] is a direct evidence that the symmetry breaking effect of the internal centrifugal force is the major driving force for the structural transition from OCT to CTBP.

On the other hand, in the CTBP isomer, when the three twisting modes are inactive having a little amount of kinetic energy, the symmetry breaking effect of the internal centrifugal force is suppressed. In this situation, the effect of the potential force becomes dominant, and as a result, it becomes highly likely that the cluster is driven from the CTBP isomer to the OCT isomer. This is because OCT has much deeper potential well than CTBP (see Fig. 1), and is much more favorable as long as the potential force is dominant. This explains why the three twisting modes tend to become highly inactive in the CTBP isomer prior to the onset of structural transition in Figs. 8(c) and 8(d). From this perspective, we expect that the major driving force for the reaction from CTBP to OCT is the potential force.

In order to demonstrate the above driving mechanisms for structural transitions more quantitatively, we present here the averaged force fields in the $a_{1}^{\prime}-a_{2}^{\prime}$ space that effectively correspond to the ones right before the onset of structural transitions. Figure 10(a) shows an averaged force field under the condition that the three twisting modes are highly active, having more than $30 \%$ of the total kinetic energy in total. This kinetic energy partitioning is based on the one at the time $t=-0.4$ before the onset of the structural transition from OCT to CTBP in Fig. 8, where the three twisting modes occupy approximately $33 \%$ of the total kinetic energy in total. Note that if the kinetic energy was equipartitioned, the three twisting modes would only have $25 \%$ of the total kinetic energy in total. Thus, the force field in Fig. 10(a) can be regarded as an effective force field right before the structural transition from OCT to CTBP. It is clearly seen from Fig. 10(a) that the force field has the strong tendency to accelerate
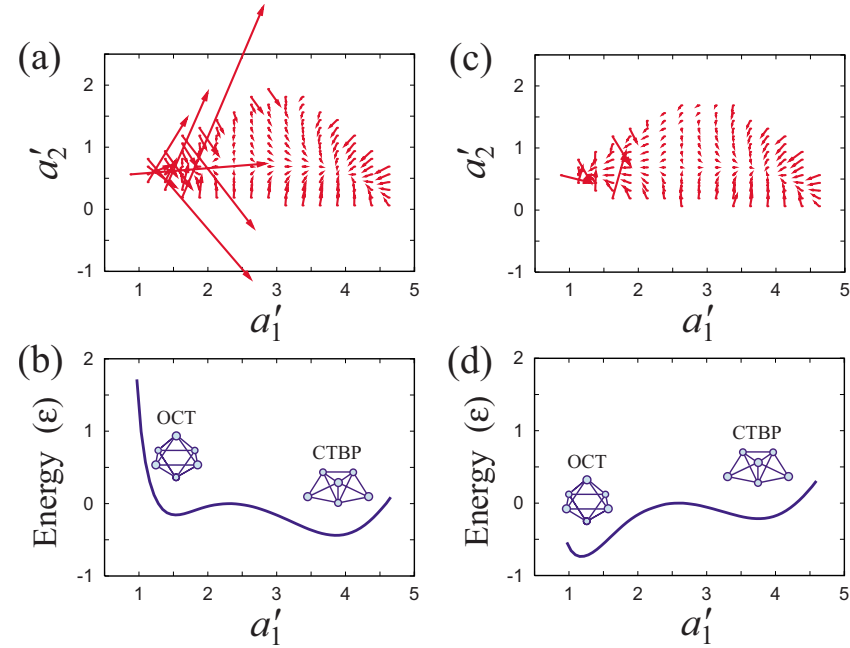

FIG. 10. (Color online) (a) Averaged force field in the space of the reaction coordinate $a_{1}^{\prime}$ and $a_{2}^{\prime}$ when the three twisting modes are highly active, having more than $30 \%$ of the total kinetic energy in total. (b) Line integral (mean force potential) of the average force field in (a) along the reaction coordinate $a_{1}^{\prime}$. (c) Average force field in the space of the reaction coordinate $a_{1}^{\prime}$ and $a_{2}^{\prime}$ when the three twisting modes are highly inactive having less than $15 \%$ of the total kinetic energy in total and the two shearing modes $\gamma_{24}$ and $\gamma_{25}$ are highly active having more than $30 \%$ of the total kinetic energy in total. (d) Line integral (mean force potential) of the average force field in (c) along the reaction coordinate $a_{1}^{\prime}$.

(drive) the system from the OCT region into the CTBP region along the reaction coordinate $a_{1}^{\prime}$. This effect can also be characterized by the corresponding mean force potential, which is shown in Fig. 10(b). This mean force potential is obtained by numerically integrating the averaged force field in Fig. 10(a) along the reaction coordinate $a_{1}^{\prime}$ in the similar way as in Fig. 9(d). The left minimum of this mean force potential corresponds to the OCT isomer, while the right one corresponds to the CTBP isomer. Figure 10(b) clearly shows that the force field in Fig. 10(a) makes the OCT less favorable and CTBP more favorable. Thus, the activation of the three twisting modes can be the principal factor to drive the structural transition from OCT to CTBP.

In a similar way, we can understand the driving mechanism for the structural transition from CTBP to OCT. Figure 10(c) shows the average force field under the conditions that the three twisting modes are highly inactive having less than $15 \%$ of the total kinetic energy in total and the two shearing modes $\gamma_{24}$ and $\gamma_{25}$ are highly active having more than $30 \%$ of the total kinetic energy in total. This kinetic energy partitioning is based on the one at time $t=-1.2$ prior to the onset of structural transition from CTBP to OCT in Fig. 8(c). In Fig. 10(c), there is no strong force field in the direction from OCT to CTBP as opposed to Fig. 10(a). This is because the symmetry breaking effect of the internal centrifugal force is suppressed due to the twisting modes being inactive. Instead, in Fig. 10(c), the potential force is dominant. As a result, this force field makes the OCT more favorable than CTBP, as can be more clearly seen from the corresponding mean force potential in Fig. 10(d). In this way, the activation and inactivation of the twisting modes switch the effective force field in the reaction coordinate and control the onset of largeamplitude collective motions. These are the main reasons 
why the three twisting modes are highly active in OCT and are highly inactive in CTBP right before structural transitions.

Finally, we note the possible roles of the two shearing modes $\gamma_{24}$ and $\gamma_{25}$, which tend to be active right before the onset of structural transition from CTBP to OCT [see Fig. 8(c)]. As can be seen from Eq. (36), these two shearing modes induce the components of the internal centrifugal force that enlarge the second gyration radius $a_{2}$. Since $a_{2}$ largely overlaps with $a_{2}^{\prime}$ [see Fig. 3(b) and the 2,2component of the matrix $\mathbf{G}$ in Eq. (30)], this effect of the $\gamma_{24^{-}}$and $\gamma_{25^{-}}$modes is characterized by a force field that is almost parallel to the positive $a_{2}^{\prime}$ axis in the $a_{1}^{\prime}-a_{2}^{\prime}$ space. Meanwhile, the isomer region (potential well) of CTBP is slightly curved into the direction of the negative $a_{2}^{\prime}$ axis from the transition region toward the bottom of the potential well, as we can see from the potential force field in Fig. 9(b). Therefore, in order for a trajectory in the vicinity of the bottom of the potential well of CTBP to get out of this well, the internal centrifugal force in the direction of the positive $a_{2}^{\prime}$ axis can also be helpful in addition to the main driving force directed to the negative $a_{1}^{\prime}$ axis. This explains why the two shearing modes $\gamma_{24}$ and $\gamma_{25}$ tend to be active right before the onset of structural transition from CTBP to OCT.

To summarize, this paper has clarified the driving mechanisms for the structural transitions of the $\mathrm{M}_{6}$ cluster in terms of the switching of the effective force field acting on the reaction coordinate. The field of the internal centrifugal force in the reaction coordinate sensitively changes depending on the amount kinetic energy in the twisting modes and the shearing modes. This is why kinetic energy needs to be partitioned in a characteristic manner among the internal modes right before structural transitions as we have seen in Fig. 8. The result of the present study can be formulated in a rather general setting as follows. In molecular reactions in which a system decreases the sphericity (symmetry) of its mass distribution, activation of the twisting modes can be the critical step that triggers the reaction. This is because the activation of the twisting modes gives rise to a strong symmetry breaking effect of the internal centrifugal force. On the other hand, in molecular reactions in which a system increases the sphericity (symmetry) of its mass distribution, inactivation of the twisting modes could generally increase the chance of the reaction as long as the spherical (symmetrical) product has lower potential energy, which is often the case in many molecules, clusters, and crystals.

\section{CONCLUDING REMARKS}

This paper has presented novel driving mechanisms for the onset of large-amplitude collective motions of atomic clusters. The key setting in which this is done is that of hyperspherical mode analysis. In this framework, the $(3 n$ -6) internal (vibrational) modes of an $n$-atom system are classified generally into three gyration-radius modes, three twisting modes, and $(3 n-12)$ shearing modes. By taking advantage of this mode analysis, the intramolecular energy transfer among the hyperspherical modes in the structural transition dynamics of a six-atom cluster has been analyzed.
It was found that a large amount of kinetic energy flows into the gyration-radius modes when the structural transitions take place. Based on this fact, a reactive mode and a corresponding reaction coordinate were constructed as a linear combination of the three gyration-radius modes. It was then found that activation or inactivation of the three twisting modes, depending on the isomer of the cluster, plays crucial roles right before the onset of structural transition. In the symmetric isomer called OCT, which has a spherical mass distribution, activation of the twisting modes initiates the structural transition into the other elongated isomer called CTBP by inducing a strong internal centrifugal force, which has an effect of elongating the mass distribution of the system. On the other hand, in the CTBP isomer, inactivation of the twisting modes initiates the structural transition into the OCT isomer by suppressing the elongation effect of the internal centrifugal force and making the effects of the potential force dominant.

The present method of hyperspherical mode analysis is in contrast to as well as being complementary to conventional normal mode analysis. While normal-mode analysis is based on the local properties of potential energy surfaces and is useful for the study of small vibrations around equilibrium points, hyperspherical mode analysis is based on global properties of the mass matrix, or metric tensor, of the internal (shape) space of $n$-atom systems and could be suitable for the study of large-amplitude motions, such as isomerization dynamics as well as dissociation and association reactions. An advantage of the hyperspherical mode analysis is that this analysis is useful for the study of intramolecular energy transfer as we have shown throughout this paper based on the concise expression of internal kinetic energy in Eq. (9). It is expected that the method of hyperspherical mode analysis can be naturally extended to the systems with nonzero angular momentum to study the energy transfer between rotational modes and internal modes. This is because the expression for the rotation-vibration kinetic energy, equation (7), is also concise, having neither cross terms nor Coriolis coupling terms.

One expects that the three gyration radii can generally serve as predominant reaction coordinates in a wide class of molecular reactions in which a system changes its mass distribution in a significant way. This expectation is based on the fact that only the gyration radii can directly control the overall mass distribution of a system. All other modes, the twisting modes and the shearing modes, by themselves cannot alter the overall mass distribution of a system directly as we noted in Sec. III. Therefore, whenever a system needs to change its overall mass distribution, a large amount of kinetic energy must flow into the gyration-radius modes. Another reason for the predominance of the gyration radii is in the fact that they are slow variables compared to other modes as was shown in Ref. 28. Thus, it will be a quite important next step to reduce the full dynamics of a large molecular system to a closed low-dimensional dynamical system of gyration radii. As we have seen in Sec. III, the dynamics of gyration radii is fairly decoupled from other bath modes in the collective motions going over the saddle regions (transition regions). On the other hand, in the dynamics in potential 
wells, gyration radii are strongly coupled with other bath modes, and this coupling is indeed essential for the onset of collective motions as we have seen in Sec. IV. Therefore, appropriate characterization of such mode coupling is crucial in order to reduce the whole dynamics of a system to the low-dimensional dynamics of gyration radii.

The roles of the twisting modes clarified in this paper can be of general interest from the viewpoint of control of molecular conformations. As we have seen in Sec. IV, the elongation effect (symmetry breaking effect) of the internal centrifugal force depends critically on the activity of the twisting modes. Therefore, if one would like to synthesize or maintain molecular conformations with a spherical mass distribution, it would be important to keep the twisting modes inactive. On the other hand, if one would like to elongate and destroy molecular conformations with a spherical mass distribution, the twisting modes of the system should be activated. From this respect, it would be interesting to study the roles of the twisting modes in the formation processes of fullerenes and viral capsids, which generally have highly symmetric and spherical mass distributions.

An important future goal is to construct a reaction rate theory by taking into consideration the driving mechanisms for molecular reactions presented in this paper. As we have noted in Sec. IV, activation or inactivation of the twisting modes serves as a "precursor" step of structural transition in each isomer. The existence of such precursor steps for reactions indicates that the reaction processes are not totally statistical but rather have a deterministic nature. If the timescale for such a precursor step is short enough compared to the average lifetime of an isomer, the reaction process of the isomer could be regarded as statistical. However, if the timescale for such precursor step is comparative to the average lifetime, the reaction processes can no longer be statistical. In the case of our $\mathrm{M}_{6}$ cluster, the timescale for intramolecular energy transfer is fast in OCT and slow in CTBP as we have noted briefly at Fig. 8, while the average lifetime of the OCT isomer and that of the CTBP isomer are comparative at the total energy of the present study $(E=-11.0 \varepsilon)$. Therefore it is expected that the nonstatistical nature becomes more prominent in the CTBP isomer than in the OCT isomer. In the future work, it will be crucially important to take into consideration such nonstatistical nature, which has so far been disregarded in conventional reaction rate theories.

To improve conventional reaction rate theories, it is useful as well as important, to clarify the phase space structures that correspond to the driving mechanisms for reactions presented in this paper. According to the dynamical system approaches, ${ }^{62-66}$ it is known that cylindrical invariant manifold "tubes" mediate the reaction dynamics going over the saddle region in the molecular phase space. In this picture, the trajectories inside the tube are essentially the reactive trajectories having sufficient kinetic energy in a reactive mode to achieve reactions. We expect that the result of Fig. 7 , which highlighted the fact that the gyration-radius reactive mode has large amount of kinetic energy in the saddle region in reactive trajectories, can be interpreted in terms of a corresponding tube structure in a coarse-grained phase space of gyration radii. Moreover, the driving mechanisms for struc- tural transitions inside potential wells presented in Sec. IV could be characterized in terms of the so-called "lobes" 71 in the coarse-grained phase space of gyration radii. An analysis of such coarse-grained phase space structures could make it possible to account for the nonstatistical reaction processes appropriately.

\section{ACKNOWLEDGMENTS}

We wish to acknowledge stimulating discussions at the Telluride Workshop on "the complexity of dynamics and kinetics in many dimensions," in which one of the authors (T.Y.) participated in 2007. In particular, we are grateful to R. Stephen Berry, Tamiki Komatsuzaki, Mikito Toda, George Haller, Sergy Grebenschikov, Laurent Wiesenfeld, Holger Waalkens, and Thomas Bartsch for their encouraging discussions. This work was partially supported by NSF Grant No. DMS-0505711. One of the authors (T.Y.) gratefully acknowledges the support from JSPS Fellowships for Research Abroad, and the research opportunity at Fukui Institute for Fundamental Chemistry, Kyoto University.

${ }^{1}$ H. Eyring, J. Chem. Phys. 3, 107 (1935).

${ }^{2}$ M. G. Evans and M. Polanyi, Trans. Faraday Soc. 31, 875 (1935).

${ }^{3}$ E. Wigner, Trans. Faraday Soc. 34, 29 (1938).

${ }^{4}$ D. G. Truhlar, B. C. Garrett, and S. J. Klippenstein, J. Phys. Chem. 100, 12771 (1996).

${ }^{5}$ W. H. Miller, J. Phys. Chem. A 102, 793 (1998).

${ }^{6}$ J. I. Steinfeld, J. S. Francisco, and W. L. Hase, Chemical Kinetics and Dynamics (Prentice-Hall, Englewood Cliffs, NJ, 1989).

${ }^{7}$ O. K. Rice and H. C. Ramsperger, J. Am. Chem. Soc. 49, 1617 (1927).

${ }^{8}$ L. S. Kassel, J. Phys. Chem. 32, 1065 (1928).

${ }^{9}$ R. A. Marcus, J. Chem. Phys. 20, 359 (1952).

${ }^{10}$ W. Forst, Unimolecular Reactions (Cambridge University Press, Cambridge, 2003).

${ }^{11}$ E. W.-G. Diau, J. L. Herek, Z. H. Kim, and A. H. Zewail, Science 279, 847 (1998).

${ }^{12}$ L. Sun, K. Song, and W. L. Hase, Science 296, 875 (2002).

${ }^{13}$ B. C. Dian, A. Longarte, and T. S. Zwier, Science 296, 2369 (2002).

${ }^{14}$ R. Mitrić, C. Bürgel, and V. Bonačić-Koutecký, Proc. Natl. Acad. Sci. U.S.A. 104, 10314 (2007).

${ }^{15}$ E. B. Wilson, Jr., J. C. Decius, and P. C. Cross, Molecular Vibrations (McGraw-Hill, New York, 1955).

${ }^{16}$ S. Sastry, Phys. Rev. Lett. 76, 3738 (1996).

${ }^{17}$ T. Keyes, J. Phys. Chem. A 101, 2921 (1997).

${ }^{18}$ B. Brooks and M. Karplus, Proc. Natl. Acad. Sci. U.S.A. 80, 6571 (1983).

${ }^{19}$ A. Kitao and N. Go, J. Comput. Chem. 12, 359 (1991).

${ }^{20}$ C. Eckart, Phys. Rev. 47, 552 (1935).

${ }^{21}$ J. D. Louck and H. W. Galbraith, Rev. Mod. Phys. 48, 69 (1976).

${ }^{22}$ T. Iwai, J. Math. Phys. 28, 964 (1987); J. Math. Phys. 28, 1315 (1987).

${ }^{23}$ R. G. Littlejohn and M. Reinsch, Phys. Rev. A 52, 2035 (1995).

${ }^{24}$ R. G. Littlejohn, K. A. Mitchell, V. A. Aquilanti, and S. Cavalli, Phys. Rev. A 58, 3705 (1998).

${ }^{25}$ R. G. Littlejohn, K. A. Mitchell, M. Reinsch, V. Aquilanti, and S. Cavalli, Phys. Rev. A 58, 3718 (1998).

${ }^{26}$ T. Yanao and K. Takatsuka, Adv. Chem. Phys. 130, 87 (2005).

${ }^{27}$ T. Yanao, W. S. Koon, and J. E. Marsden, Phys. Rev. A 73, 052704 (2006).

${ }^{28}$ T. Yanao, W. S. Koon, J. E. Marsden, and I. G. Kevrekidis, J. Chem. Phys. 126, 124102 (2007).

${ }^{29}$ C. Eckart, Phys. Rev. 46, 383 (1934).

${ }^{30}$ X. Chapuisat and A. Nauts, Phys. Rev. A 44, 1328 (1991).

${ }^{31}$ X. Chapuisat, Phys. Rev. A 45, 4277 (1992).

${ }^{32}$ A. Kuppermann, Adv. Mol. Vib. Collision Dyn. 2B, 117 (1993).

${ }^{33}$ A. Kuppermann, J. Phys. Chem. 100, 2621 (1996).

${ }^{34}$ A. Kuppermann, J. Phys. Chem. 101, 6368 (1997).

${ }^{35}$ V. Aquilanti, A. Lombardi, and E. Yurtsever, Phys. Chem. Chem. Phys. 4, 5040 (2002). 
${ }^{36}$ V. Aquilanti, A. Lombardi, M. B. Sevryuk, and E. Yurtsever, Phys. Rev. Lett. 93, 113402 (2004).

${ }^{37}$ V. Aquilanti, A. Lombardi, and M. B. Sevryuk, J. Chem. Phys. 121, 5579 (2004).

${ }^{38}$ M. B. Sevryuk, A. Lombardi, and V. Aquilanti, Phys. Rev. A 72, 033201 (2005).

${ }^{39}$ M. Kummer, Indiana Univ. Math. J. 30, 281 (1981).

${ }^{40}$ J. E. Marsden, R. Montgomery, and T. Ratiu, Contemp. Math. 28, 101 (1984).

${ }^{41}$ J. E. Marsden and T. S. Ratiu, Introduction to Mechanics and Symmetry (Springer, New York, 1999).

${ }^{42}$ J. E. Marsden, T. S. Ratiu, and J. Scheurle, J. Math. Phys. 41, 3379 (2000)

${ }^{43}$ A. M. Bloch, Nonholonomic Mechanics and Control (Springer, New York, 2003)

${ }^{44}$ A. Guichardet, Ann. Inst. Henri Poincare 40, 329 (1984).

${ }^{45}$ T. Iwai, Ann. Inst. Henri Poincare 47, 199 (1987).

${ }^{46}$ A. Tachibana and T. Iwai, Phys. Rev. A 33, 2262 (1986).

${ }^{47}$ R. G. Littlejohn and M. Reinsch, Rev. Mod. Phys. 69, 213 (1997).

${ }^{48}$ R. S. Berry, Chem. Rev. (Washington, D.C.) 93, 2379 (1993).

${ }^{49}$ P. A. Braier, R. S. Berry, and D. J. Wales, J. Chem. Phys. 93, 8745 (1990).

${ }^{50}$ J. Jellinek, T. L. Beck, and R. S. Berry, J. Chem. Phys. 84, 2783 (1986)

${ }^{51}$ H. L. Davis, J. Jellinek, and R. S. Berry, J. Chem. Phys. 86, 6456 (1987).

${ }^{52}$ T. Komatsuzaki and R. S. Berry, J. Chem. Phys. 110, 9160 (1999); 115, 4105 (2001).

${ }^{53}$ K. Fukui, J. Phys. Chem. 74, 4161 (1970)

${ }^{54}$ W. H. Miller, N. C. Handy, and J. E. Adams, J. Chem. Phys. 72, 99 (1980).

${ }^{55}$ M. Page and J. W. McIver, Jr., J. Chem. Phys. 88, 922 (1988).
${ }^{56}$ P. G. Bolhuis, D. Chandler, C. Dellago, and P. L. Geissler, Annu. Rev. Phys. Chem. 53, 291 (2002).

${ }^{57}$ G. Hummer, J. Chem. Phys. 120, 516 (2004).

${ }^{58}$ R. B. Best and G. Hummer, Proc. Natl. Acad. Sci. U.S.A. 102, 6732 (2005).

${ }^{59}$ W. E, W. Ren, and E. Vanden-Eijnden, Chem. Phys. Lett. 413, 242 (2005).

${ }^{60}$ W. E and E. Vanden-Eijnden, J. Stat. Mech.: Theory Exp. 123, 503 (2006).

${ }^{61}$ P. Metzner, C. Schütte, and E. Vanden-Eijnden, J. Chem. Phys. 125, 084110 (2006).

${ }^{62}$ N. De Leon, M. A. Mehta, and R. Q. Topper, J. Chem. Phys. 94, 8310 (1991).

${ }^{63}$ T. Uzer, C. Jaffé, J. Palacián, P. Yanguas, and S. Wiggins, Nonlinearity 15, 957 (2002)

${ }^{64}$ H. Waalkens, A. Burbanks, and S. Wiggins, J. Chem. Phys. 121, 6207 (2004).

${ }^{65}$ F. Gabern, W. S. Koon, J. E. Marsden, and S. D. Ross, Physica D 211, 391 (2005).

${ }^{66}$ C. B. Li, Y. Matsunaga, M. Toda, and T. Komatsuzaki, J. Chem. Phys. 123, 184301 (2005).

${ }^{67}$ G. Hummer and I. G. Kevrekidis, J. Chem. Phys. 118, 10762 (2003).

${ }^{68}$ D. I. Kopelevich, A. Z. Panagiotopoulos, and I. G. Kevreidis, J. Chem. Phys. 122, 044907 (2005); J. Chem. Phys. 122, 044908 (2005).

${ }^{69}$ A. Kitao, S. Hayward, and N. Go, Proteins 33, 496 (1998).

${ }^{70}$ T. Komatsuzaki, K. Hoshino, Y. Matsunaga, G. J. Rylance, R. L. Johnston, and D. J. Wales, J. Chem. Phys. 122, 084714 (2005).

${ }^{71}$ M. J. Davis and S. K. Gray, J. Chem. Phys. 84, 5389 (1986).

${ }^{72}$ T. Yanao and K. Takatsuka, Phys. Rev. A 68, 032714 (2003).

${ }^{73}$ T. Yanao and K. Takatsuka, J. Chem. Phys. 120, 8924 (2004). 